\title{
PARP inhibition enhances tumor cell-intrinsic immunity in ERCC1-deficient non-small cell lung cancer
}

\author{
Roman M. Chabanon, ${ }^{1,2,3,4}$ Gareth Muirhead, ${ }^{3}$ Dragomir B. Krastev, ${ }^{3,4}$ Julien Adam, ${ }^{2}$ Daphné Morel, ${ }^{1,2}$ Marlène Garrido, ${ }^{2}$ Andrew Lamb, ${ }^{5}$ \\ Clémence Hénon, ${ }^{1,2}$ Nicolas Dorvault, ${ }^{2}$ Mathieu Rouanne, ${ }^{1,6}$ Rebecca Marlow, ${ }^{7}$ Ilirjana Bajrami, ${ }^{3,4}$ Marta Llorca Cardeñosa, ${ }^{3,4,8}$ \\ Asha Konde, ${ }^{3,4}$ Benjamin Besse, ${ }^{1,9}$ Alan Ashworth, ${ }^{10}$ Stephen J. Pettitt, ${ }^{3,4}$ Syed Haider, ${ }^{3}$ Aurélien Marabelle, ${ }^{6,11}$ Andrew N.J. Tutt, ${ }^{3,7}$ \\ Jean-Charles Soria, ${ }^{1}$ Christopher J. Lord, ${ }^{3,4}$ and Sophie Postel-Vinay, ${ }^{1,2,11}$ \\ 'Université Paris Saclay, Université Paris-Sud, Faculté de médicine, Le Kremlin Bicêtre, Paris, France. ${ }^{2}$ ATIP-Avenir group, Inserm U981, Gustave Roussy, Villejuif, France. ${ }^{3}$ The Breast Cancer Now Toby Robins \\ Breast Cancer Research Centre and ${ }^{4}$ CRUK Gene Function Laboratory, The Institute of Cancer Research, London, United Kingdom. ${ }^{5}$ Sage Bionetworks, Seattle, Washington, USA. ${ }^{6}$ Inserm U1015, Gustave \\ Roussy, Villejuif, France. ${ }^{7}$ The Breast Cancer Now Research Unit, King's College London, London, United Kingdom. ${ }^{8}$ Biomedical Research Institute INCLIVA, Hospital Clinico Universitario Valencia, University \\ of Valencia, Valencia, Spain. ${ }^{9}$ Department of Medical Oncology, Gustave Roussy, Villejuif, France. ${ }^{10}$ UCSF Helen Diller Family Comprehensive Cancer Center, San Francisco, California, USA. "Département \\ d'Innovations Thérapeutiques et Essais Précoces (DITEP), Gustave Roussy, Villejuif, France.
}

\begin{abstract}
The cyclic GMP-AMP synthase/stimulator of IFN genes (cGAS/STING) pathway detects cytosolic DNA to activate innate immune responses. Poly(ADP-ribose) polymerase inhibitors (PARPi) selectively target cancer cells with DNA repair deficiencies such as those caused by BRCA1 mutations or ERCC1 defects. Using isogenic cell lines and patient-derived samples, we showed that ERCC1-defective non-small cell lung cancer (NSCLC) cells exhibit an enhanced type I IFN transcriptomic signature and that low ERCC1 expression correlates with increased lymphocytic infiltration. We demonstrated that clinical PARPi, including olaparib and rucaparib, have cell-autonomous immunomodulatory properties in ERCC1-defective NSCLC and BRCA1-defective triple-negative breast cancer (TNBC) cells. Mechanistically, PARPi generated cytoplasmic chromatin fragments with characteristics of micronuclei; these were found to activate cCAS/STING, downstream type I IFN signaling, and CCL5 secretion. Importantly, these effects were suppressed in PARP1-null TNBC cells, suggesting that this phenotype resulted from an on-target effect of PARPi on PARP1. PARPi also potentiated IFN- $\gamma$-induced PD-L1 expression in NSCLC cell lines and in fresh patient tumor cells; this effect was enhanced in ERCC1-deficient contexts. Our data provide a preclinical rationale for using PARPi as immunomodulatory agents in appropriately molecularly selected populations.
\end{abstract}

\section{Introduction}

Immune checkpoint inhibitors (ICIs) have revolutionized the prognosis of several aggressive cancers, notably non-small cell lung cancer (NSCLC). Recent impressive results of large phase III trials in NSCLC have reported unprecedented improvements in overall survival and progression-free survival when anti-programmed death receptor 1 or anti-programmed death ligand 1 (antiPD-[L]1) was used in first-line therapy $(1,2)$. Likewise, remarkable 5 -year survival rates of $16 \%$ have recently been reported in this disease (3), highlighting the ability of these agents to provide longterm tumor control. Although these results are encouraging, they also reinforce the fact that still only a minority of patients receive long-term benefit. Better understanding of the determinants of

\footnotetext{
Conflict of interest: SPV has received research funding from Merck KGaA for an unrelated project, as part of the funding for her research team. AA, CJL, and ANJT are named inventors on patents (US patent nos. 9611223, 8143241) describing the use of PARPi and stand to gain from their use as part of the Institute of the Cancer Research "Rewards to Inventor" scheme. JCS has been a full-time employee of Medimmune/AstraZeneca since September 2017. He has received consultancy fees from AstraZeneca, Roche, Sanofi, Servier, and Pierre Fabre. AM has received consultancy fees and honoraria from Roche/Genentech, Pfizer, Novartis, Lytix Biopharma, Bristol-Myers Squibb, and MSD. License: Copyright 2019, American Society for Clinical Investigation.

Submitted: July 3, 2018; Accepted: December 18, 2018.

Reference information: J Clin Invest. 2019;129(3):1211-1228.

https://doi.org/10.1172/JCl123319.
}

response to ICIs and identification of rational combinations that would increase the proportion of patients benefiting from these therapies are therefore crucial.

Several factors have been associated with response to immunotherapy: tumor-related factors (e.g., cancer cell mutations), microenvironment-related factors (e.g., expression of immune checkpoints, lymphocytic infiltration, or IFN signatures), and host-related factors (e.g., microbiome) (4). Defects in the DNA damage response (DDR) in cancer cells are key determinants of cancer immunogenicity. Indeed, DDR defects result in genomic instability and increased tumor mutational burden (TMB), which has been linked - at least in some cases - to better outcome upon ICI treatment (5). The best illustrations of this are probably mismatch repair-deficient tumors (6) and POLE/POLD1-mutated endometrial carcinoma and glioblastoma $(7,8)$, which are highly sensitive to ICIs, likely due to their increased neoantigen repertoire. High TMB has also been correlated with better response to ICIs in melanoma (9) and $\operatorname{NSCLC}(2,10,11)$. Other DNA repair defects, such as BRCA1/2 mutations, have been found to be enriched in ICI responders (12). However, a simple correlation among DNA repair defect-induced genomic instability, TMB, and response to ICIs cannot be claimed (5), as tumor heterogeneity (13) and other determinants of response also play a role that, importantly, seems to be independent from TMB in response to ICIs $(14,15)$. 
A ZFN targeting ERCC1

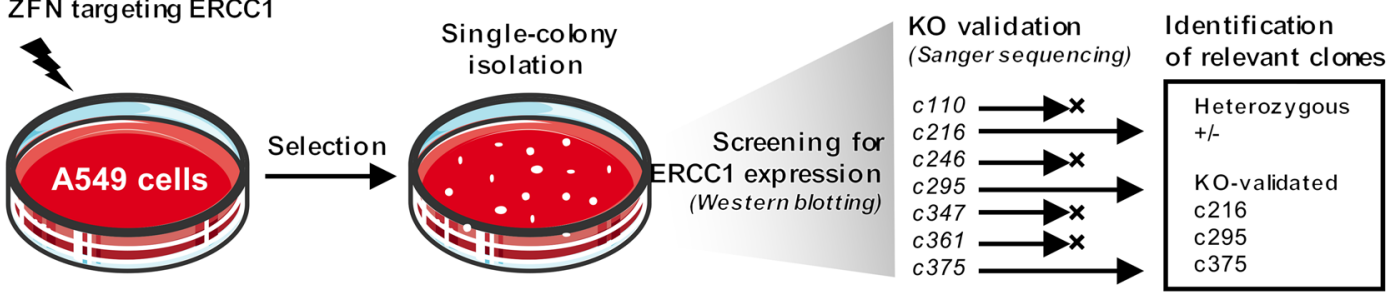

B
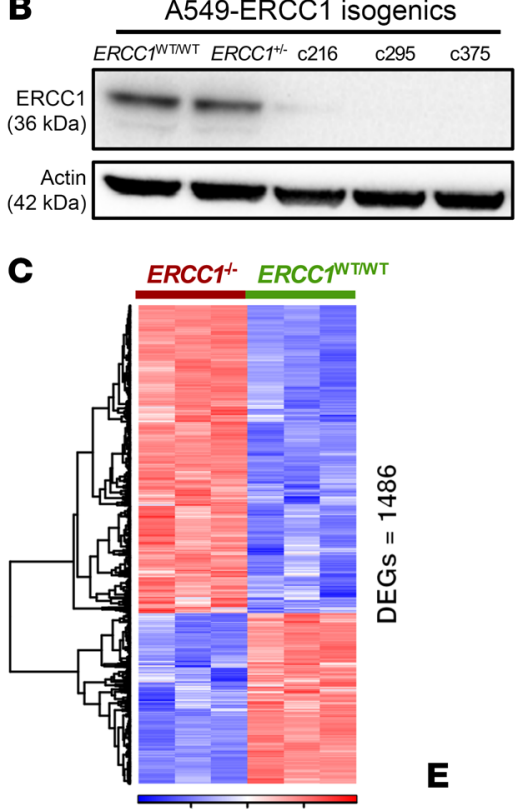

E
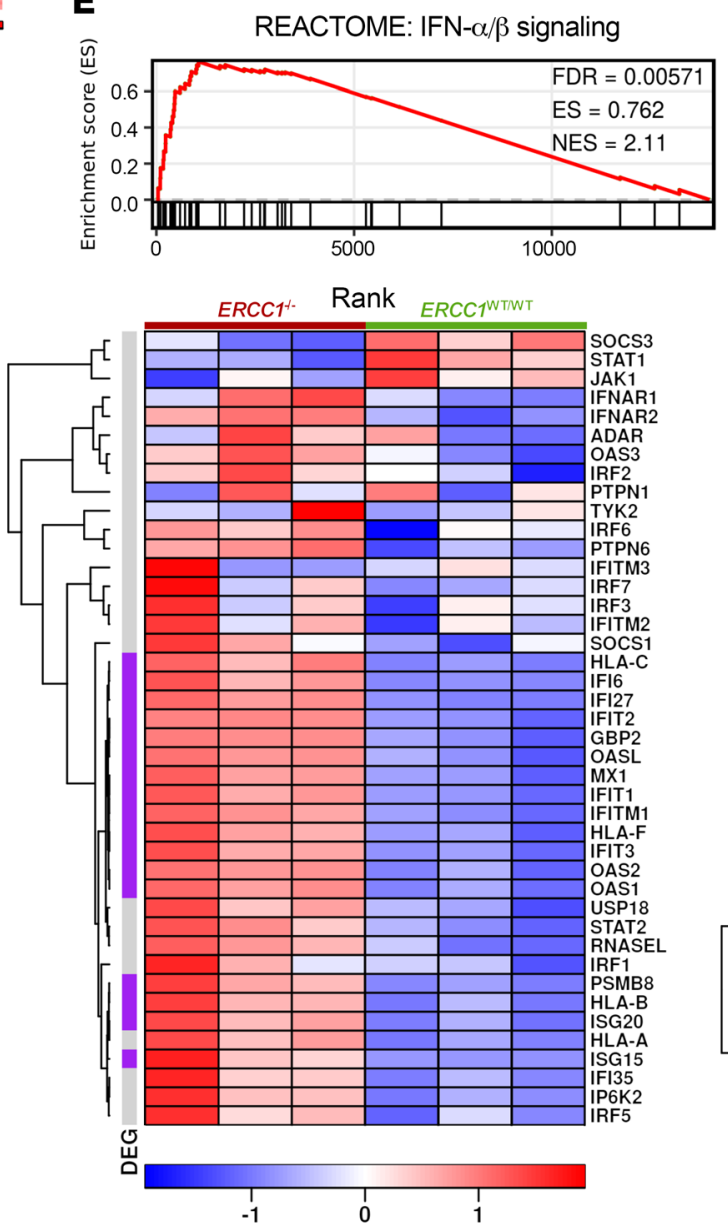

REACTOME pathways
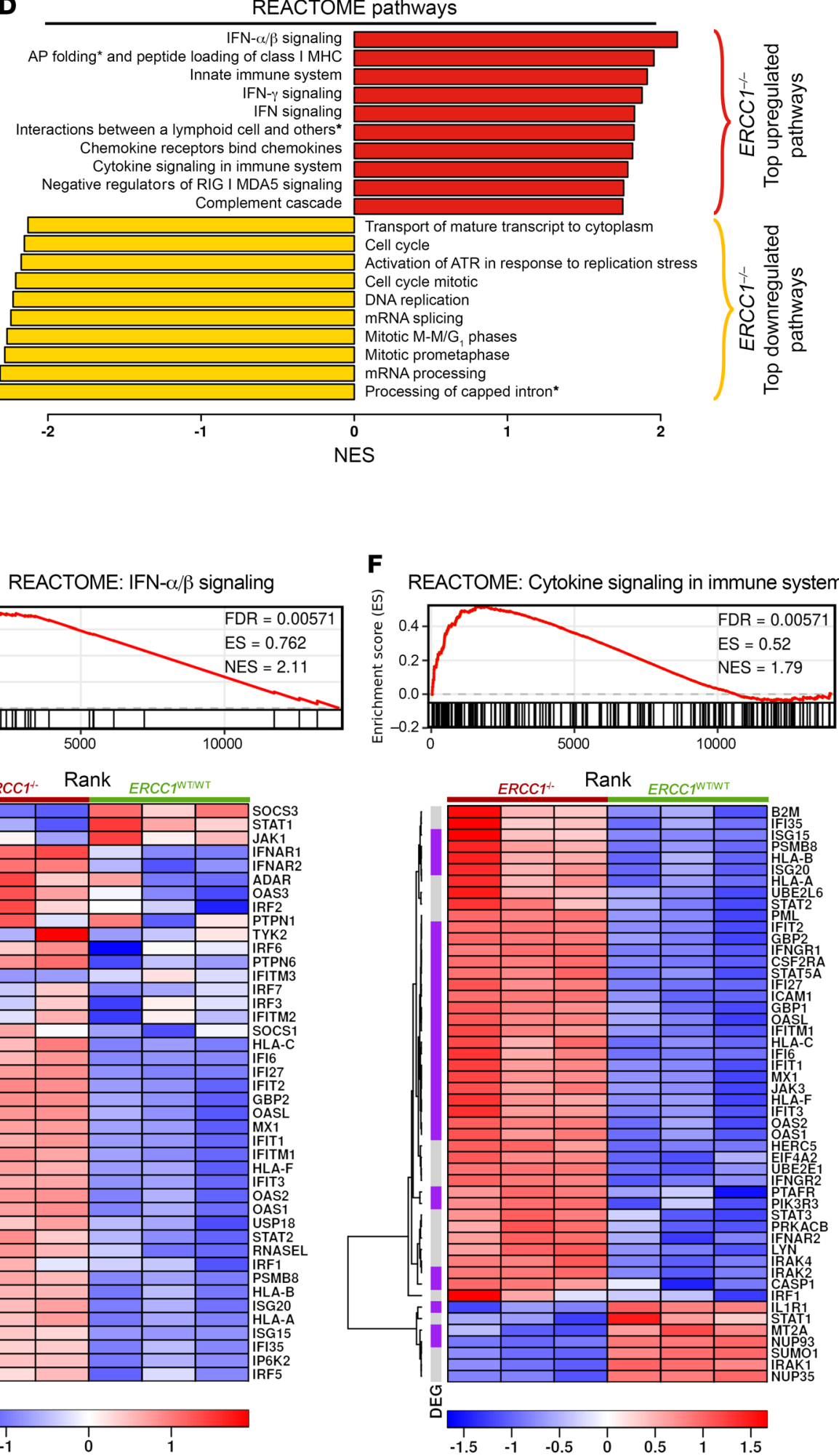
Figure 1. Loss of ERCC1 results in increased type I IFN and cytokine signaling in NSCLC models in vitro. (A) Schematic of the generation of ERCC1-deficient clones from the parental NSCLC cell line A549. Full procedures are detailed in Friboulet et al. (31). (B) Western blot showing expression of ERCC1 in the parental (ERCC1 $\left.{ }^{\mathrm{WT} / \mathrm{WT}}\right)$, heterozygous $\left(\mathrm{ERCC} 1^{+-}\right.$), and ERCC1-knockout clones (c216, c295, and c375). (C) Heatmap displaying all significantly differentially expressed genes (significantly DEGs) in A549-ERCC1 ${ }^{-1-}$ cells compared with A549-ERCC $1^{\text {WT/WT }}$ cells, determined by RNA-Seq. $n=3$; heatmap scale is a $Z$ score. Threshold for differential expression was $|\mathrm{LFC}|>1$, and threshold for significance was FDR $<$ 0.05. (D) GSEA of REACTOME pathways in A549-ERCC1-1- compared with A549-ERCC1 ${ }^{\text {WT/WT }}$ cells. Red, top 10 upregulated REACTOME pathways in A549-ERCC1-1- cells; yellow, top 10 downregulated REACTOME pathways in A549-ERCC1 ${ }^{-/-}$cells. All pathways displayed had FDR $<0.05$. AP folding*, antigen presentation folding assembly; Processing of capped intron*, processing of capped intron containing pre-mRNA; Interactions between a lymphoid cell and others*, interaction between a lymphoid cell and non-lymphoid cells. (E) GSEA of the REACTOME pathway "IFN- $\alpha / \beta$ signaling," and associated heatmap showing the genes of the pathway, ranked by FDR. $n=3$; heatmap scale is a $Z$ score. (F) GSEA of the REACTOME pathway "Cytokine signaling in immune system," and associated heatmap showing the genes of the pathway, ranked by FDR. $n=3$; heatmap scale is a $Z$ score. In $\mathbf{E}$ and $\mathbf{F}$, purple, significantly DEGs with FDR $<0.05$ and $|L F C|$ $>1$; gray, nonsignificantly DEGs.

Another interface between DDR and immunogenicity that has recently generated particular attention in immuno-oncology is the cyclic GMP-AMP synthase/stimulator of IFN genes (cGAS/ STING) pathway (16). This pathway, involved in the sensing of foreign or damaged cytosolic DNA, triggers innate immune responses through the activation of a signaling cascade connecting the cytoplasmic DNA sensor cGAS, several signal transducers including STING and TBK1, and eventually transcription factors (mainly IRF3 and $\mathrm{NF}-\mathrm{kB}$ ) that are collectively responsible for the induction of a type I IFN response (16). Thus, processes that disrupt nuclear DNA integrity and favor the translocation of DNA to the cytosol (either in the context of endogenous DNA repair deficiency or through the use of exogenous DNA-damaging agents) may activate cGAS/ STING. For example, defects in homologous recombination (HR) genes (BRCA1/2 or FANCD2) and use of radiotherapy or S phasedependent chemotherapies have been associated with cell cycledependent formation of cytoplasmic chromatin fragments (CCFs) and subsequent increase in STING signaling in breast cancer (1720). Further, cGAS recently appeared to be an essential mediator of the antitumor effects of ICIs (21), and identification of cGAS/ STING activators has become an area of intense research, with several ongoing phase I trials evaluating such molecules (22). As one of the main therapeutic challenges in immuno-oncology is currently to turn "cold," non-T cell-inflamed tumors into "hot," T cell-inflamed tumors, a better understanding of the cellular contexts in which targeted therapies can activate the cGAS/STING pathway is key.

DDR-deficient cancers that present elevated genomic instability may represent a favorable environment for selective cGAS/ STING activation. Excision repair cross-complementation group 1 (ERCC1) deficiency is the most frequent DDR defect in NSCLC and occurs in $30 \%-50 \%$ of cases (23). The tumor suppressor protein BRCA1 is also frequently defective in triple-negative breast cancer (TNBC), following either germline mutation or somatic alteration (mutation or epigenetic silencing) $(24,25)$. Both ERCC1 and $B R C A 1$ defects confer sensitivity to platinum-based therapy
$(26,27)$ and PARP inhibitors (PARPi) $(28,29)$, and while PARPi have demonstrated their efficacy in advanced BRCA-deficient breast cancers (30), these agents are also being clinically assessed in ERCC1-defective (platinum-sensitive) NSCLC (PIPSeN trial, NCT02679963). Therefore, ERCC1 deficiency represents an attractive candidate for harnessing CGAS/STING activation in NSCLC, where ICIs have shown unprecedented efficacy, yet in only a small proportion of patients.

Here, we show that loss of ERCC1 in NSCLC leads to increased STING expression and constitutive activation of type I IFN signaling, which associates with enhanced $\mathrm{T}$ cell infiltration in patient-derived samples. Using a unique combination of isogenic models of ERCC1-deficient NSCLC, BRCA1-deficient and PARPi-resistant TNBC, we find that multiple clinical PARPi generate cytosolic DNA in a cell cycle- and DDR defect-dependent fashion, as a result of an on-target effect of PARPi. This in turn activates cGAS/STING signaling and elicits specific tumor cell-intrinsic immune responses, including type I IFN response and CCL5 secretion. PARPi further synergize with IFN- $\gamma$ to induce cell surface PD-L1 expression in NSCLC models, a phenotype that is specifically enhanced in ERCC1-deficient cells. Our data reveal an unexpected immunomodulatory potential of PARPi that could be therapeutically exploited to enhance ICI efficacy in ERCC1deficient NSCLC patients.

\section{Results}

ERCC1 deficiency in isogenic systems is associated with increased type I IFN signaling, cytokine signaling, and lymphocytic infiltration in NSCLC. We hypothesized that lack of function of a key DNA repair tumor suppressor gene, such as ERCC1, in tumor cells, might influence the molecular processes that control antitumor cell immune responses. To address this hypothesis in a relatively unbiased fashion, we first used RNA-Seq to profile the transcriptome of isogenic ERCC1-defective and WT A549 NSCLC cells (31, 32). Briefly, this isogenic model was generated using zinc finger targeting of ERCC1, and consists of one A549-ERCC $1^{\mathrm{WT} / \mathrm{WT}}$ parental cell line; one ERCC1-heterozygous cell line (herein referred to as A549-ERCC1 ${ }^{+-}$); and $3 \mathrm{ERCC1}^{-/-}$clones, in which we reconfirmed no detectable levels of ERCC1 (Figure 1, A and B) and which are characterized by exquisite sensitivity to cisplatin (31) - highlighting their clinical relevance (26) - and to PARPi (28) (Supplemental Figure 1A). Comparative transcriptomics of A549-ERCC1 ${ }^{\mathrm{WT} / \mathrm{WT}}$ and one $\mathrm{ERCC1}^{-1-}$ clone (c216, referred to as A549-ERCC1-/- below) revealed 1486 significantly differentially expressed genes (DEGs; Figure 1C). Consistent with the known functions of the ERCC1/ XPF endonuclease in the resolution of stalled replication fork and subsequent appropriate cell cycle progression, gene set enrichment analysis (GSEA) using the REACTOME pathway database revealed significant downregulation of several DNA repair-, cell cycle-, and DNA replication-related pathways in A549-ERCC1-- cells (Figure 1D and Supplemental Figure 1B). This analysis also identified significant enrichment of numerous immune-related pathways in A549-ERCC1 ${ }^{-/}$cells - indeed, 24 immune-related pathways were found among the top 50 upregulated REACTOME terms (Supplemental Figure 1C) - suggesting a role for ERCC1 in modulating the immune characteristics of NSCLC cells in a cell-autonomous fashion. Among these pathways, the most significantly enriched were 
A

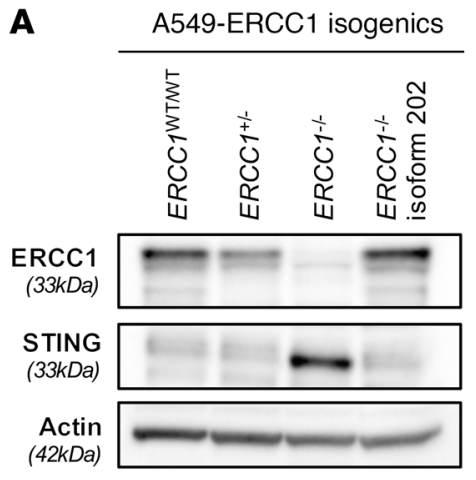

Actin

(42kDa)

C

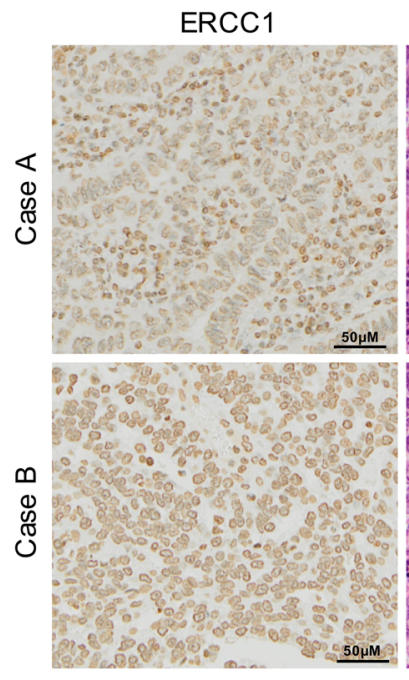

B

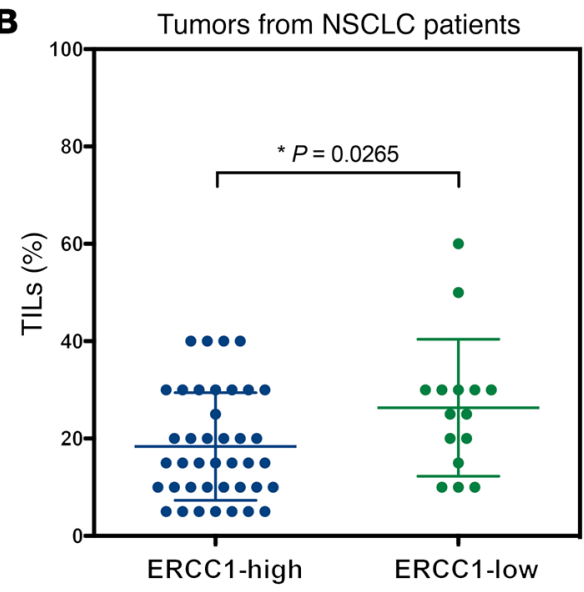

H\&E

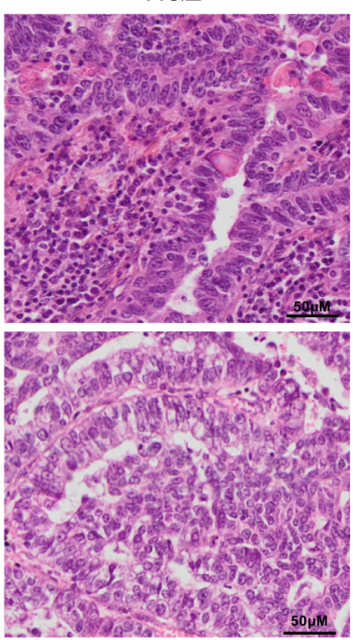

Figure 2. Loss of ERCC1 associates with increased STING expression in vitro and enhanced lymphocytic infiltration in human NSCLC samples.

(A) Western blot illustrating ERCC1 and STING expression in A549-ERCC1 ${ }^{\text {WT/WT }}$, A549-ERCC1 ${ }^{+/}$, A549-ERCC1 ${ }^{-1-}$ and A549-ERCC1 ${ }^{-1-}+$ isoform 202 isogenic cell lines. (B) Scatter box plots of ERCC1 protein expression (assessed by IHC staining) and the percentage of TILs (assessed through morphology) in a series of resected human NSCLC adenocarcinoma samples $(n=55)$. Tumors were classified according to the expression of ERCC1, and the corresponding level of TILs was plotted for each individual tumor. Mann-Whitney $U$ test. (C) Representative images of ERCC1 and H\&E stainings in 2 surgical specimens of resected lung adenocarcinoma. Case A shows low ERCC1 staining in tumor cells and high stromal TIL density; case B shows high ERCC1 staining in tumor cells and low stromal TIL density. Scale bars: $50 \mu \mathrm{m}$. type I and II IFN signaling, antigen presentation through class I major histocompatibility complex (MHC-I), and innate immune system and cytokine signaling (Figure 1D). Interpretation of GSEA plots confirmed that type I IFN signaling was significantly enriched in ERCC1-deficient cells compared with isogenic WT cells (normalized enrichment score $[\mathrm{NES}]=2.11$; FDR $=0.0057$ ) (Figure $1 \mathrm{E})$. Consistent with this enrichment, refined analysis of the transcriptomic data also identified several IFN- $\alpha / \beta / \gamma$-inducible proteins as being upregulated in ERCC1-defective cells, including several members of the STAT family (Supplemental Figure 1D). Cytokine signaling was also found to be significantly enriched in the A549-ERCC1 ${ }^{-/}$cell line $(\mathrm{NES}=1.79 ; \mathrm{FDR}=0.0057$ ) (Figure $1 \mathrm{~F})$, and consistent with this result, we found an upregulation of several C-C motif and C-X-C motif chemokines in those cells (Supplemental Figure 1E). In particular, the chemotactic chemokines CCL2, CCL5, CXCL1, CXCL2, CXCL5, CXCL8, and CXCL10 were upregulated greater than 5 -fold in A549-ERCC1 ${ }^{-/}$cells $(F D R<$ 0.05). Considering the critical immunogenic and lymphoattractant properties of these chemokines (33), this suggested that ERCC1 deficiency might contribute to the establishment of cancer cellautonomous immunity in NSCLC cells. These findings were also obtained in 2 independent A549-ERCC1 ${ }^{-/-}$clones (c295 and c375), which showed similar significant enrichment in type I IFN signaling on RNA-Seq profiling (NES $=2.54, \mathrm{FDR}=0.0062$ for $\mathrm{c} 295$ and
$\mathrm{NES}=2.79, \mathrm{FDR}=0.0063$ for c375; Supplemental Figure 2, A-D). As ERCC1-deficient NSCLC tumors still harbor residual levels of ERCC1, we sought to investigate which threshold of ERCC1 deficiency was sufficient to induce such a type I IFN signature in vitro by using the ERCC1-heterozygous cell line of our isogenic model (A549-ERCC1 ${ }^{+/-}$cell line). Interestingly, the type I IFN signature was also significantly enriched in this model, albeit to a lesser extent than in $E R C C 1^{-1-}$ clones (NES = 1.86; FDR = 0.0131; Supplemental Figure 2, E and F). Whole exome sequencing of the isogenic clones did not identify mutations in any gene involved in immune signaling (28), suggesting the defect in ERCC1 as the most likely cause of the observed transcriptional dysregulation.

In other contexts, DDR defects have been linked to innate immune response and type I IFN signaling via cytosolic DNA sensing, notably involving the cGAS/STING pathway $(16,17)$. We hypothesized that loss of ERCC1 might modulate such signals. Although expression of STING, the major initiator of the cytosolic DNA-sensing pathway, was almost undetectable by Western blotting in A549-ERCC1 ${ }^{\text {WT/WT }}$ and A549-ERCC1 ${ }^{+/-}$cells, we found elevated STING protein expression in A549-ERCC1 ${ }^{-/}$cells (Figure 2A). Consistent with this, STING mRNA levels were increased more than 2.5-fold in A549-ERCC1 ${ }^{-/-}$cells ( $\log _{2}$ fold change [LFC] $=1.3769, \mathrm{FDR}=0.0009)$, compatible with a transcriptional mode of regulation. A similar upregulation of STING was also detected 
A

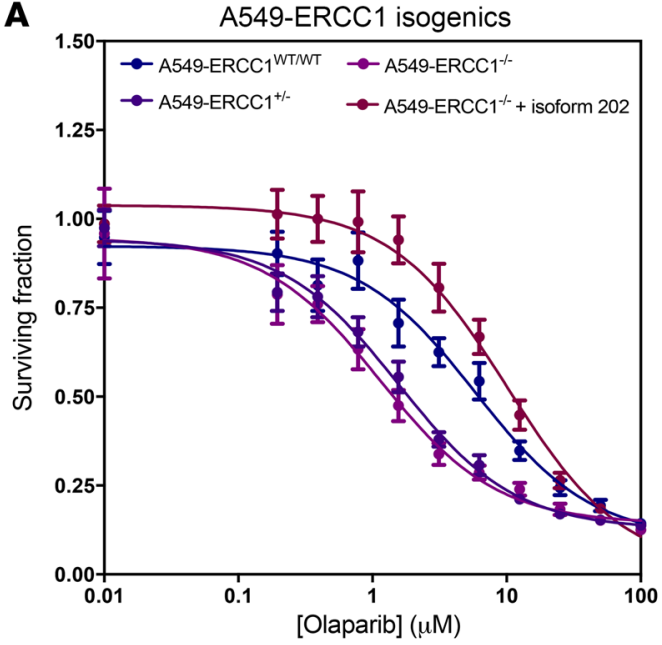

C
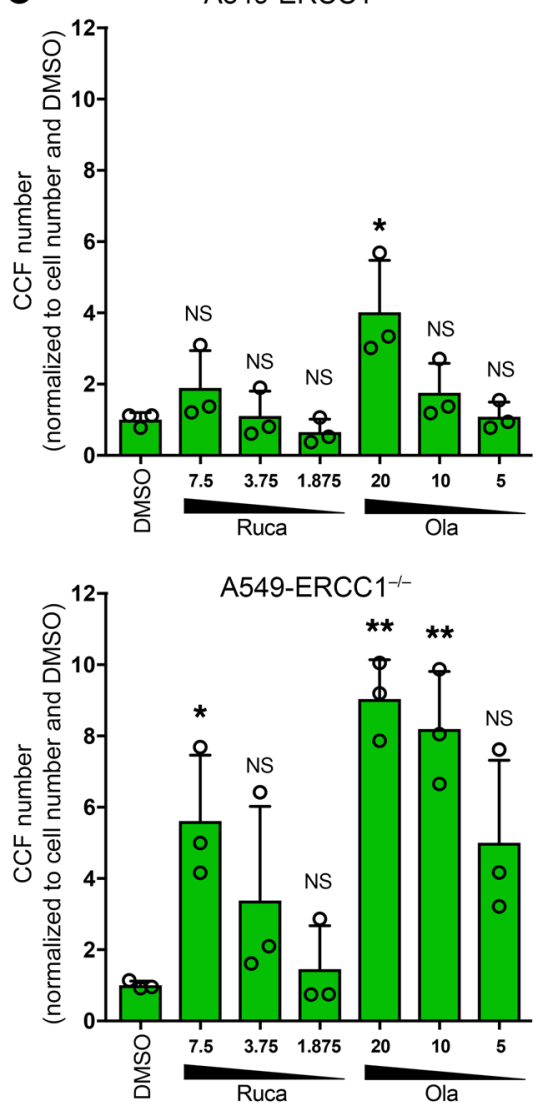

A549-ERCC $1^{+/-}$
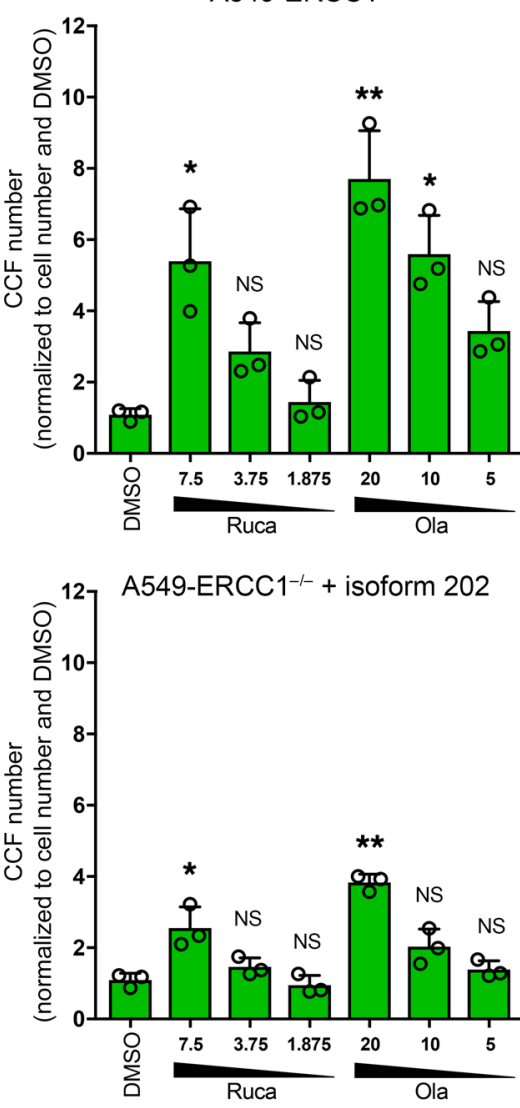

B

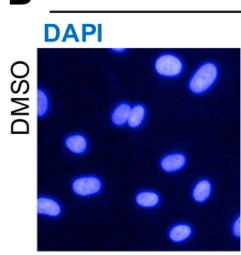

A549-ERCC1 1wTNT
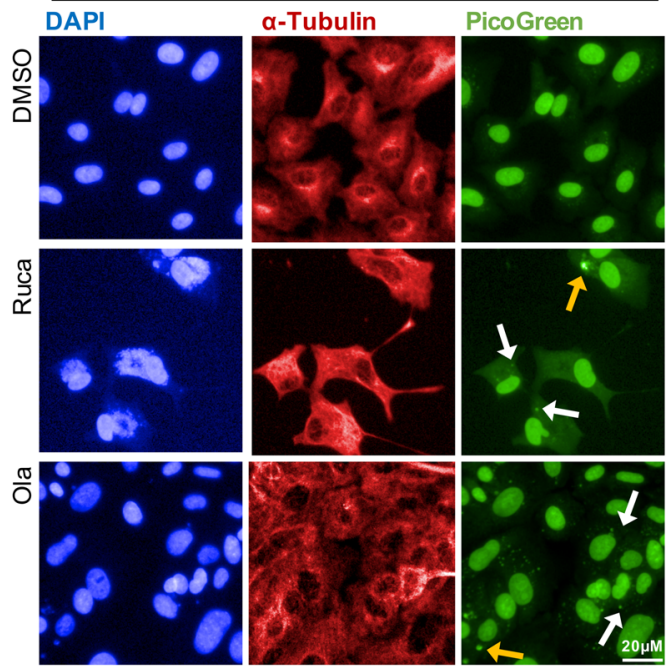

A549-ERCC1-1-

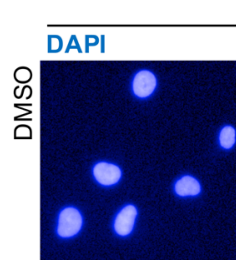

a-Tubulin

\section{PicoGreen}
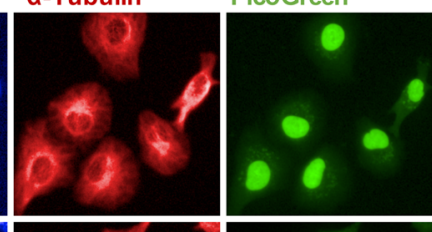

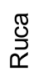
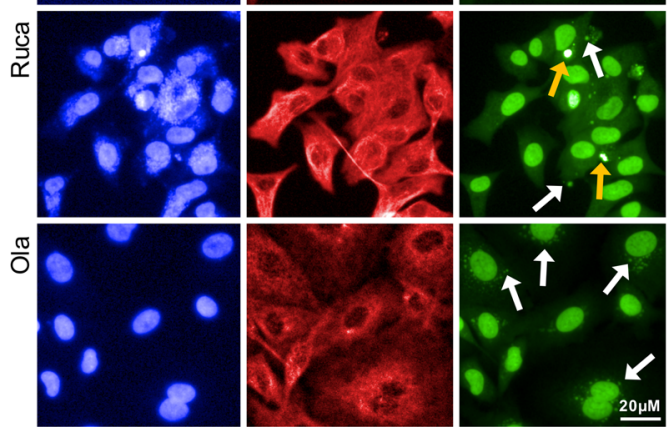

in the 2 other A549-ERCC1-deficient clones (Supplemental Figure $2 \mathrm{G}$ ). In order to confirm that this observation was a primary effect of ERCC1 deficiency, we assessed STING expression in an isogenic cell line derived from the A549-ERCC1 ${ }^{-/}$clone in which the functional isoform of ERCC1 (isoform 202) had been stably
Figure 3. PARPi generate CCFs in an ERCC1-dependent manner in NSCLC cells. (A) Assessment of olaparib (Ola) cytotoxicity in A549-ER$\mathrm{CC}^{\mathrm{WT} / \mathrm{WT}}$ versus A549-ERCC1+/-, A549-ERCC1 ${ }^{-1-}$, and A549-ERCC1 ${ }^{-/-}+$isoform 202 cell lines. Cells were treated with a range of doses of Ola and continuously exposed to the drug for 5 days. Shown are dose-response curves showing surviving fractions; mean $\pm S D, n=4$. (B) Representative immunofluorescence images of DMSO-, rucaparib- (Ruca-), and Olaexposed A549-ERCC1 ${ }^{\text {WT/WT }}$ and A549-ERCC1 ${ }^{-1-}$ cells. Cells were exposed to $15 \mu \mathrm{M}$ Ruca or $40 \mu \mathrm{M}$ Ola during 72 hours. White arrows, CCFs; yellow arrows, micronuclei. Scale bar: $20 \mu \mathrm{m}$. (C) Automated quantification of CCFs in A549-ERCC1 isogenic cells exposed to increasing doses of Ruca or Ola $(\mu \mathrm{M})$. Shown is CCF number per cell normalized to DMSO. Mean \pm $\mathrm{SD}, n=3$; ${ }^{*} P<0.05,{ }^{*} P<0.01$, Kruskal-Wallis test and post hoc Dunn's test, relative to DMSO control. Results shown are representative of 2 experiments performed with similar results.

reintroduced (herein referred to as "A549-ERCC1 ${ }^{-1-}$ + isoform 202 "). Strikingly, reexpression of ERCC1 in this isogenic model led to loss of STING protein expression (Figure 2A), suggesting ERCC1-dependent reversible modulation of STING expression. Of note, cGAS expression was also increased in A549-ERCC1 ${ }^{-/-}$ 
A CRISPR/Cas9
Single-colony isolation
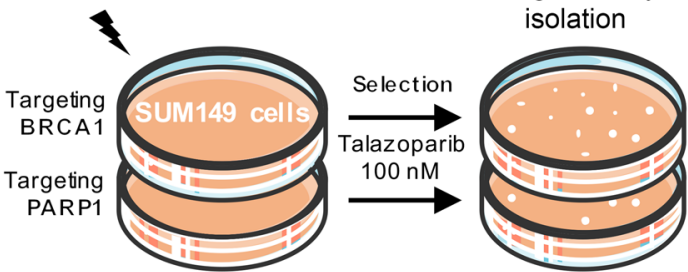

Genotype validation Identification

(Sanger sequencing) of relevant clones

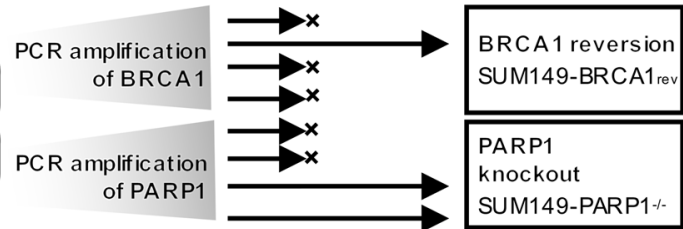

SUM149-PARP1-1-

B

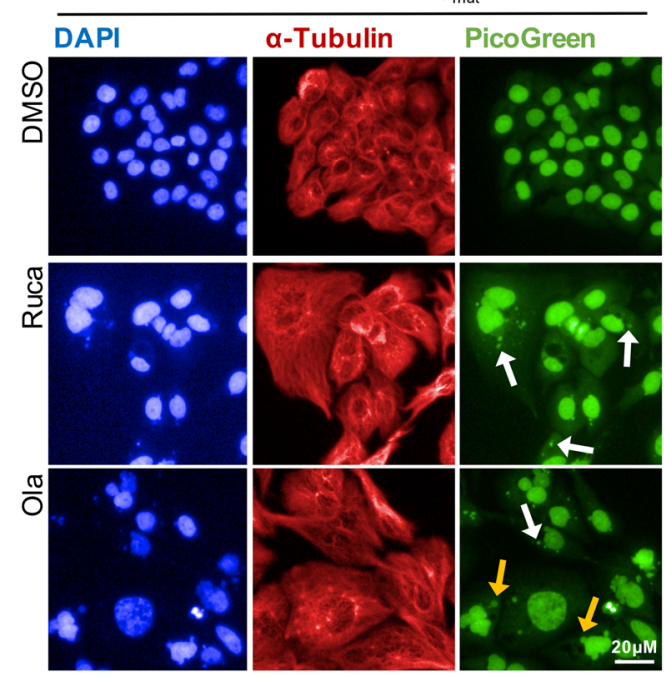

DAPI $\alpha$-Tubulin PicoGreen
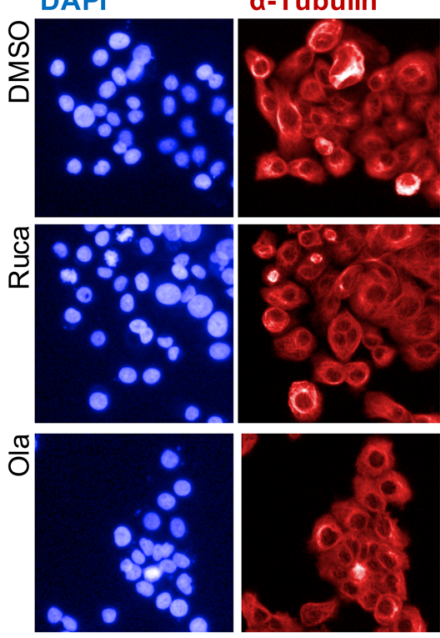

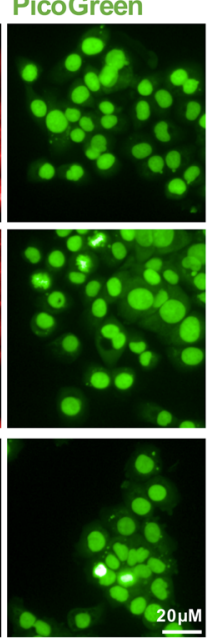

C

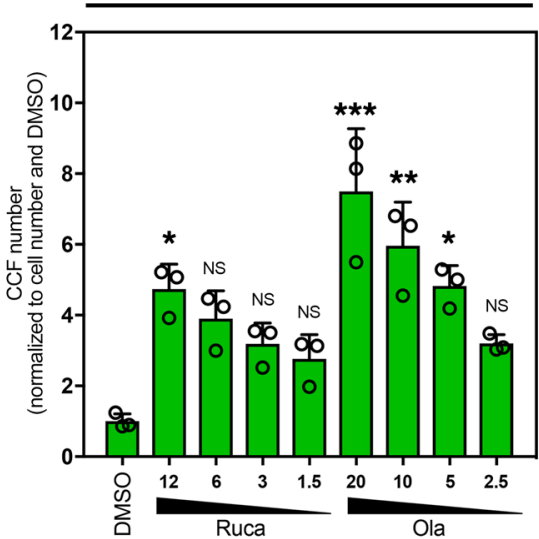

SUM149-BRCA1 $1_{\text {rev }}$

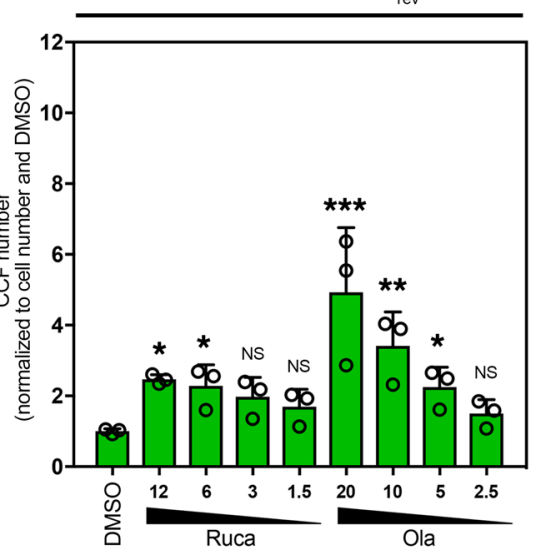

E

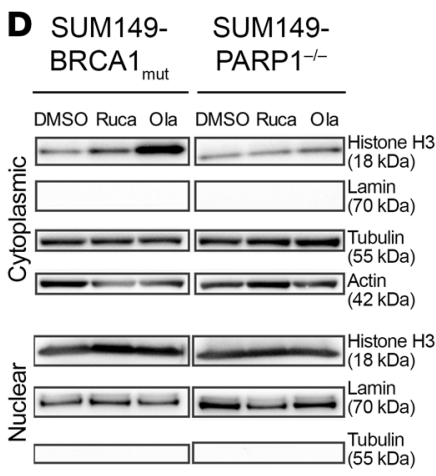

A549-ERCC1 1 wT/WT

E

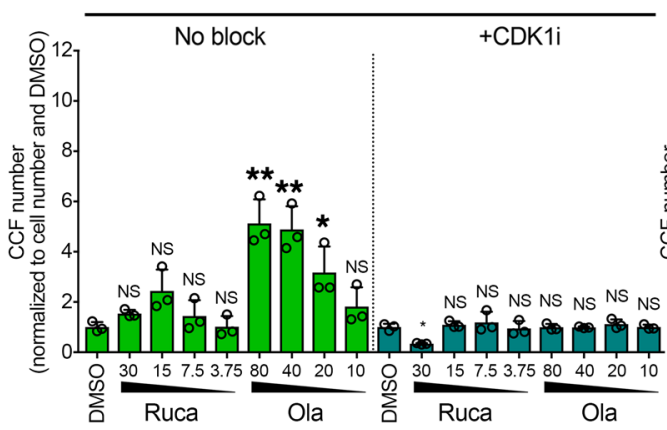

SUM149-PARP1-1-
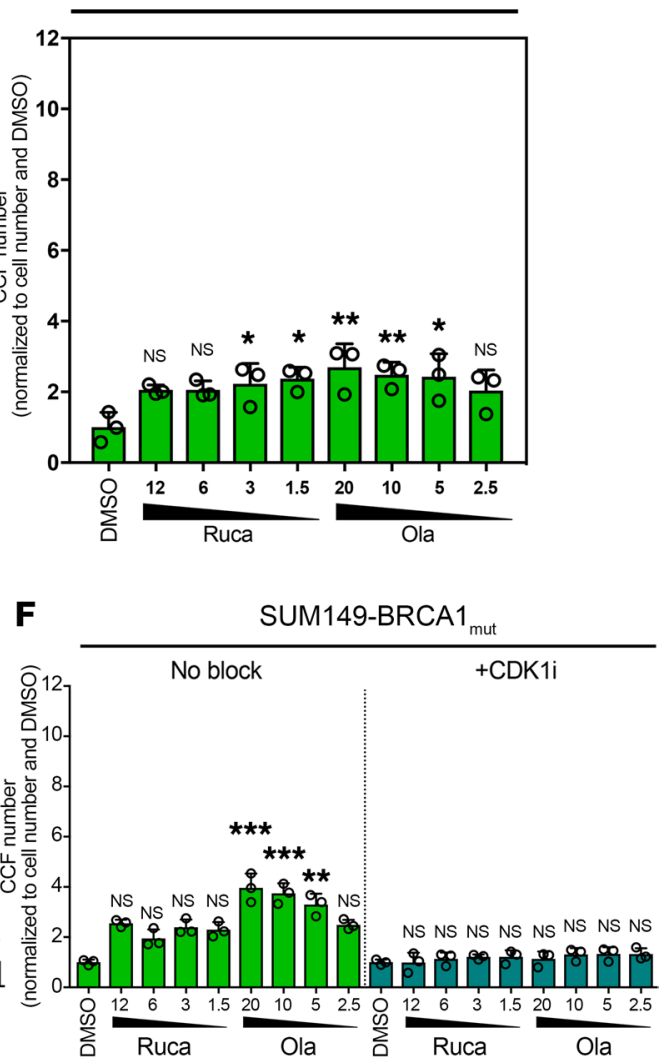
Figure 4. PARPi generate CCFs in a DNA repair defect- and cell cycledependent manner. (A) Schematic of the generation of BRCA1-revertant and PARP1-knockout cell lines from the parental BRCA1-mutant SUM149 TNBC cell line. (B) Representative immunofluorescence images of DMSO-, Ruca-, and Ola-exposed SUM149-BRCA1 ${ }_{\text {mut }}$ and SUM149-PARP1-/- cells. Cells were exposed to $6 \mu \mathrm{M}$ Ruca, $10 \mu \mathrm{M}$ Ola, or DMSO (vehicle) during 72 hours. White arrows, CCFs; yellow arrows, micronuclei. Scale bars: $20 \mu \mathrm{m}$. (C) Automated quantification of CCFs in SUM149-BRCA $1_{\text {mut }}$, SUM149BRCA1 $_{\text {rev }}$, and SUM149-PARP1 ${ }^{-/-}$cells exposed to increasing doses of Ruca or Ola $(\mu \mathrm{M})$. Shown is CCF number per cell normalized to DMSO. Mean \pm SD, $n=3$, Kruskal-Wallis test and post hoc Dunn's test, relative to DMSO control. Results shown are representative of 2 experiments performed with similar results. (D) Western blot of histone $\mathrm{H} 3$ in the nuclear and cytoplasmic fractions of SUM149-BRCA $1_{\text {mut }}$ and SUM149-PARP1 ${ }^{-1-}$ cells exposed to PARPi during 48 hours. $\beta$-Tubulin and lamin B1 were used as fraction purity controls. (E and $\mathbf{F}$ ) Automated quantification of CCFs in A549-ERCC1 ${ }^{\text {WT/WT }}(\mathbf{E})$ and SUM149-BRCA ${ }_{\text {mut }}$ (F) cells exposed to increasing doses of Ruca or Ola $(\mu \mathrm{M})$ in the presence or absence of the cell cycle blocker CDK1i RO-3306. Shown is CCF number per cell normalized to DMSO. Mean $\pm \mathrm{SD}, n=3$, Kruskal-Wallis test and post hoc Dunn's test, relative to DMSO control. ${ }^{*} P<0.05,{ }^{* *} P<0.01,{ }^{* * *} P<0.001$.

cells $(\mathrm{LFC}=0.5336, \mathrm{FDR}=0.0098)$. As activation of cGAS/ STING results in phosphorylation of TBK1, recruitment of IRF3, and eventually expression of type I IFN genes (16), this suggested that the observed transcriptomic profile could be linked to STING activation in A549-ERCC1 $1^{-/}$cells.

In order to evaluate whether this enhanced cell-autonomous immune signaling could shape the tumor microenvironment, we estimated the extent of tumor-infiltrating lymphocytes (TILs) in a series of 55 human tumor samples derived from patients with resected lung adenocarcinoma (stages I, II, and IIIA). ERCC1 status in these tumors was evaluated by IHC as previously described (32), and TILs were assessed using a morphology-based coverage score. This analysis identified a statistically significant association between low ERCC1 expression and high levels of TILs ( $P=$ 0.0265, Mann-Whitney $U$ test, Figure 2, B and C).

Taken together, these data suggest that ERCC1 loss results in a constitutive and cell-autonomous increase in STING expression and type I IFN signaling in NSCLC models in vitro. These observations are consistent with enhanced tumor lymphocytic infiltration in vivo in human NSCLC adenocarcinoma samples.

PARPi generate CCFs in an ERCC1 defect- and cell cycledependent manner. As previously reported (28), A549-ERCC1 ${ }^{-1}$ cells show enhanced sensitivity to PARPi when compared with A549-ERCC $1^{\mathrm{WT} / \mathrm{WT}}$ cells, an effect that we reconfirmed in shortterm survival assays (Figure 3A). We further hypothesized that PARPi - which not only inhibit the catalytic activity of PARP1, but also trap PARP1 onto the DNA, causing stalled replication forks and subsequent double-strand breaks (DSBs) (34) - might specifically favor the formation of CCFs and in turn trigger cGAS/STING signaling in ERCC1-defective tumor cells.

To test this hypothesis, we exposed A549-ERCC1 isogenic cell lines to increasing concentrations of 2 different clinical PARPi, rucaparib and olaparib, and monitored the presence of CCFs using immunofluorescence. For each PARPi, we selected concentrations surrounding the SF50 (dose generating 50\% cell survival in shortterm assays, after 5 days of exposure to the drug) of the parental cell line; we therefore started with a dose corresponding to the SF80 of the WT cell line and applied a 2 -fold increase at each subsequent dose to determine the final dose range. Although the number of CCFs in A549-ERCC1 ${ }^{\text {WT/WT }}$, A549-ERCC1 ${ }^{+/}$, A549-ERCC1 ${ }^{-/}$, and A549-ERCC1 ${ }^{--}+$isoform 202 cells was not significantly different in the absence of PARPi (Supplemental Figure 3A), we observed a dose-dependent increase in CCF number upon PARPi exposure, an effect that was significantly enhanced in A549-ERCC1 ${ }^{-/-}$and A549-ERCC1 ${ }^{+/-}$models compared with A549-ERCC1 ${ }^{\text {WT } / \text { WT }}$ cells (Figure 3, B and C; $>6$-fold difference at $10 \mu \mathrm{M}$ olaparib, $P=$ 0.0016 ; $>4$-fold difference at $5 \mu \mathrm{M}$ olaparib, $P=0.03$; 2-way ANOVA, post hoc Šidák's test), but not in A549-ERCC1 ${ }^{-/-}+$isoform 202 cells. These findings were further validated in the independent H1975-ERCC1 isogenic NSCLC model: in these cells, ERCC1 loss caused increased CCF levels in the absence of treatment (Supplemental Figure 3A; 2.5-fold increase, $P=0.0035$, Welch's $t$ test), a phenotype that was enhanced upon PARPi exposure (Supplemental Figure 3, B and C).

BRCA1-defective tumor cells are also profoundly sensitive to PARPi (29). We therefore assessed whether the effects seen in ERCC1-defective cells could be reproduced in BRCA1-defective cells. To do this, we used recently described isogenic series derived from the BRCA1-mutant TNBC SUM149 cell line $(35,36)$. This isogenic series consists of 3 cell lines (Figure 4A): one BRCA1-mutant parental line (SUM149-BRCA1 ${ }_{\text {mut }}$ ); one BRCA1-mutant reverted line that is PARPi-resistant following restoration of the native $B R C A 1$ reading frame and functionality (35) (SUM149-BRCA1 ${ }_{\text {rev }}$ ); and one SUM149 clone with PARPi resistance caused by loss of PARP1 (36) (SUM149-PARP1-/-). In the absence of PARPi, SUM149-BRCA1 ${ }_{\text {mut }}$ cells showed a significantly higher number of CCFs compared with SUM149-BRCA1 cells (>8-fold increase, $P=0.0233$, Welch's $t$ test; Supplemental Figure 3A). Upon PARPi exposure, we observed a dose-dependent increase in CCF number in SUM149-BRCA1 ${ }_{\text {mut }}$ cells, but not in SUM149-PARP1 ${ }^{-/-}$cells, confirming the on-target effect of PARPi in triggering this phenotype (Figure 4, B and C); SUM149BRCA1 $1_{\text {rev }}$ cells showed an intermediate phenotype (Figure $4 \mathrm{C}$ ), confirming that the generation of CCFs was a BRCA1-dependent process. As PARPi have been reported to modulate histone PARylation and chromatin structure (37), thereby potentially promoting transcription of retroelements, we further confirmed the presence of chromatin (i.e., histone-bound DNA as opposed to naked DNA) by assessing the presence of histone $\mathrm{H} 3$ in cytoplasmic fractions. This demonstrated increased H3 levels after PARPi exposure in SUM149-BRCA1 ${ }_{\text {mut }}$ cells, but not in SUM149-PARP1 $1^{--}$cells, thus confirming, respectively, the presence of cytoplasmic chromatin and the on-target effect of PARPi (Figure 4D). Consistent with this finding, assessment of H3K27me3 by immunofluorescence in the A549-ERCC1 isogenic cells revealed colocalization of this heterochromatin marker with CCFs in PARPi-exposed cells (Supplemental Figure 3D).

As PARPi cause $\mathrm{S}$ phase-dependent DNA damage, we hypothesized that the observed CCFs might, at least in part, be micronuclei $(19,20)$. Micronuclei are signs of genomic or chromosomal instability which have two main characteristics: their formation is cell cycle dependent, as they arise during anaphase from lagging chromosomes or chromatid bridges following unresolved DNA lesions; and they contain heterochro- 

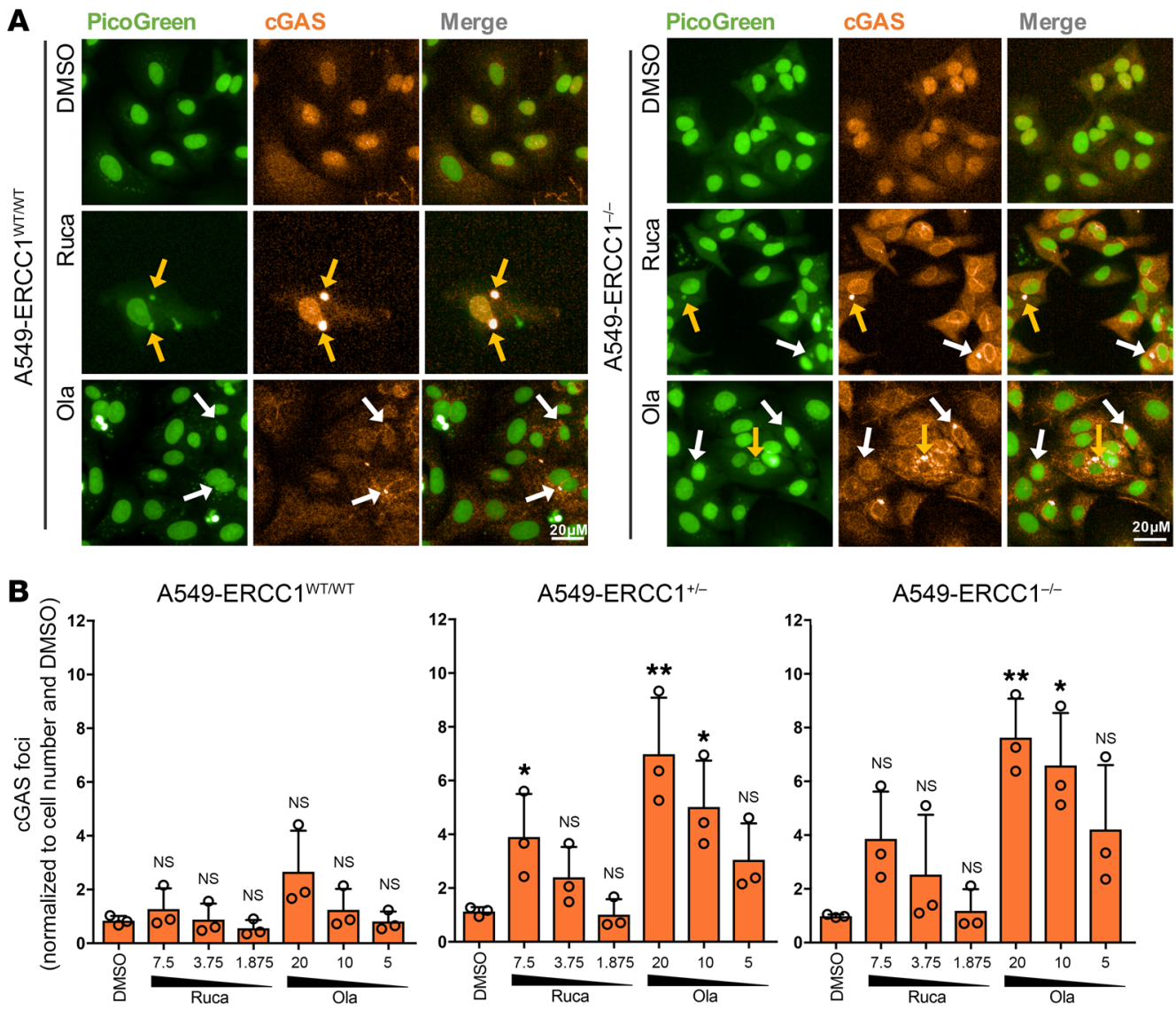

A549-ERCC $1^{+/-}$

A549-ERCC $1^{-1-}$
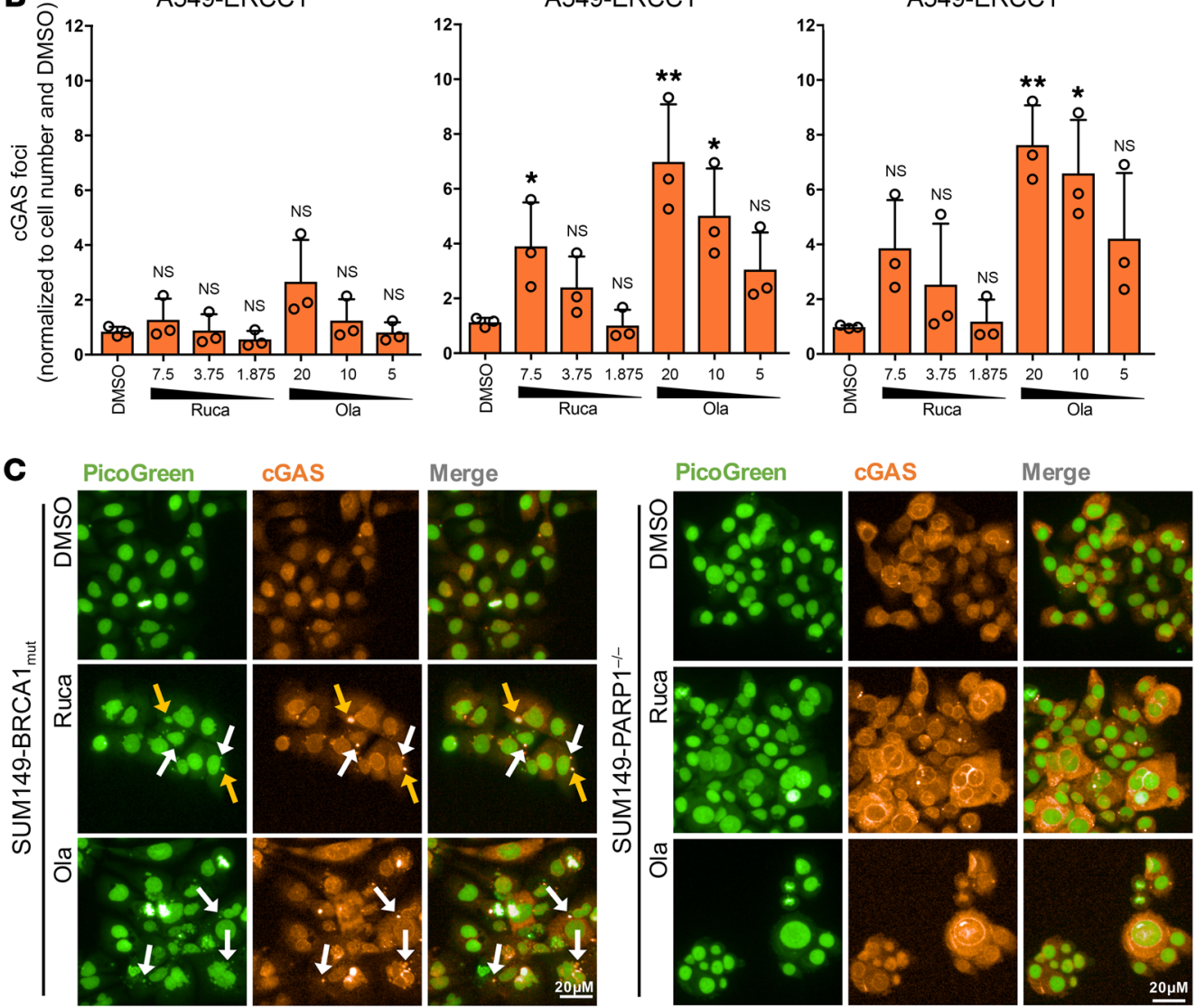

E

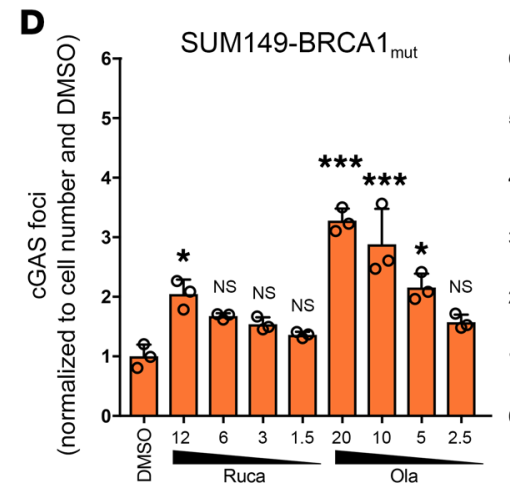

SUM149-BRCA1 ${ }_{\text {rev }}$
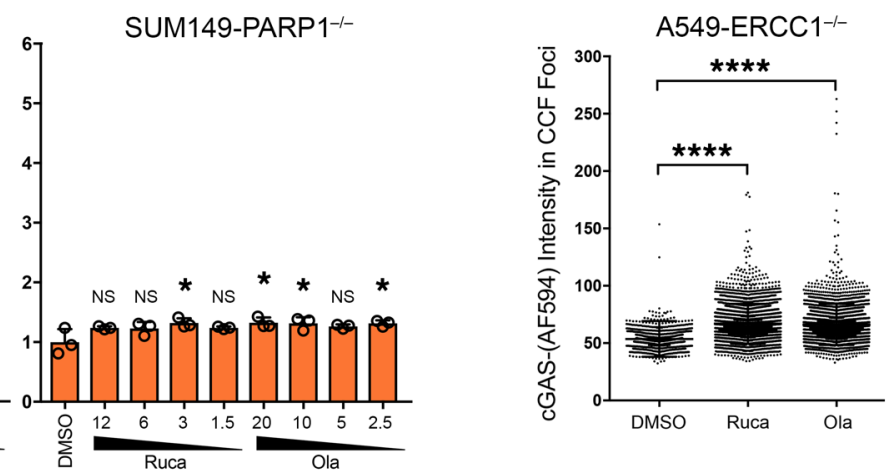
Figure 5. PARPi-induced CCFs are detected by cGAS. (A) Representative immunofluorescence images of DMSO-, Ruca-, and Ola-exposed A549ERCC1 ${ }^{\mathrm{WT} / \mathrm{WT}}$ and A549-ERCC1 ${ }^{-1-}$ cells. Cells were exposed to $15 \mu \mathrm{M}$ Ruca or $40 \mu \mathrm{M}$ Ola during 72 hours. White arrows, CCFs; yellow arrows, micronuclei. Scale bars: $20 \mu \mathrm{m}$. Images corresponding to the DMSO condition originate from the same image field as those shown in Figure 3B. (B) Automated quantification of cytoplasmic cGAS foci in A549-ERCC1 isogenic cells exposed to increasing doses of Ruca or Ola $(\mu \mathrm{M})$. Shown are numbers of cytoplasmic cGAS foci per cell normalized to DMSO. Mean \pm SD, $n=3$, Kruskal-Wallis test and post hoc Dunn's test, relative to DMSO control. Results shown are representative of 2 experiments performed with similar results. (C) Representative immunofluorescence images of DMSO-, Ruca-, and Ola-exposed SUM149-BRCA $1_{\text {mut }}$ and SUM149-PARP1 ${ }^{-1-}$ cells. Cells were exposed to $6 \mu \mathrm{M}$ Ruca, $10 \mu \mathrm{M}$ Ola, or DMSO (vehicle) during 72 hours. White arrows, CCFs; yellow arrows, micronuclei. Scale bars: $20 \mu \mathrm{m}$. (D) Automated quantification of cytoplasmic cGAS foci in SUM149-BRCA1 ${ }_{\text {mut' }}$, SUM149-BRCA1 ${ }_{\text {rev }}$, and SUM149$\mathrm{PARP}^{-1-}$ cells exposed to increasing doses of Ruca or Ola ( $\left.\mu \mathrm{M}\right)$. Shown are numbers of cytoplasmic cGAS foci per cell normalized to DMSO. Mean \pm SD, $n=3$; Kruskal-Wallis test and post hoc Dunn's test, relative to DMSO control. Results shown are representative of 2 experiments performed, with similar results. (E) Scatter box plots displaying cCAS foci intensity for each colocalizing CCF foci in A549-ERCC1 ${ }^{\text {WT/WT }}$ and A549-ERCC1-1- cells exposed to DMSO (vehicle), $15 \mu \mathrm{M}$ Ruca, or $40 \mu \mathrm{M}$ Ola. $n=3$, Kruskal-Wallis test and post hoc Dunn's test. ${ }^{*} P<0.05,{ }^{* *} P<0.01,{ }^{* * *} P<0.001,{ }^{* * *} P<0.0001$.

matin, initially surrounded by a fragile lamin B1 membrane that is eventually easily ruptured (19). We therefore first assessed the formation of CCFs in the presence of PARPi and upon cell cycle blockade in A549-ERCC1 ${ }^{\mathrm{WT} / \mathrm{WT}}$ cells, using 3 different cell cycle inhibitors: (i) the CDK1 inhibitor (CDK1i) RO-3306; (ii) hydroxyurea; and (iii) 5-fluorouracil. All 3 cell cycle inhibitors caused a complete abrogation of the PARPi-induced generation of CCFs, supporting that their formation was cell cycle dependent (Figure 4E and Supplemental Figure 3, E and F). Similar observations were made in the SUM149-BRCA1 ${ }_{\text {mut }}$ cell line (Figure 4F). Immunofluorescence analysis of the costaining of H3K27me3 and lamin B1 in the A549-ERCC1 isogenic model revealed some structures typical of micronuclei (Supplemental Figure 3D), although the fragile lamin B1 envelope was not detectable around all PicoGreen/H3K27me3-colocalizing foci. Automated quantification of micronuclei revealed baseline patterns similar to that of CCFs (Supplemental Figure 3A), as well as a consistent dose-dependent formation of micronuclei upon PARPi exposure, in both ERCC1- and BRCA1-isogenic models (Supplemental Figure 3, G and H). Consistent with our previous observations (Figure $3 \mathrm{C}$ and Figure $4 \mathrm{C}$ ), this effect was more pronounced in A549-ERCC1 ${ }^{-/}$(Supplemental Figure 3G) and SUM149-BRCA1 ${ }_{\text {mut }}$ cells (Supplemental Figure $3 \mathrm{H}$ ) and abrogated in the SUM149-PARP1 ${ }^{-/-}$model.

These data support the hypothesis that PARPi exposure promotes the accumulation of CCFs, some of which have characteristics of micronuclei. This phenotype results from an on-target effect of PARPi, is cell cycle dependent, and is enhanced in ERCC1-defective NSCLC cells and other PARPi-sensitive models such as BRCA1-defective TNBC.
A

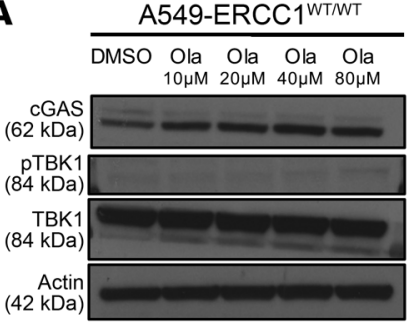

A549-ERCC1 ${ }^{-1-}$

DMSO Ola Ola Ola Ola

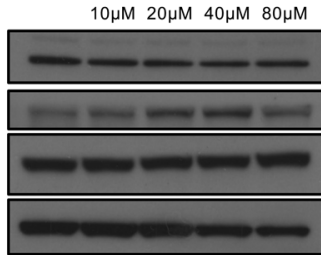

B

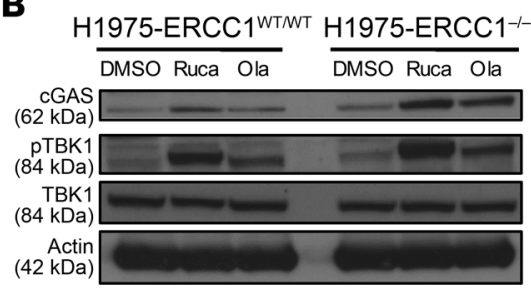

C H1975-ERCC1 1WTNT

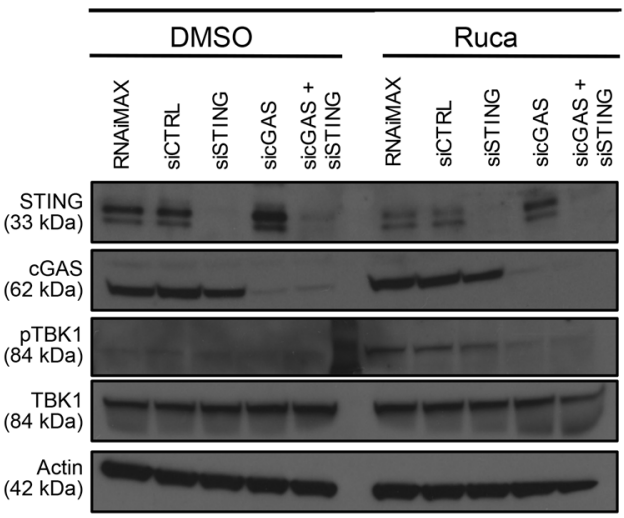

D

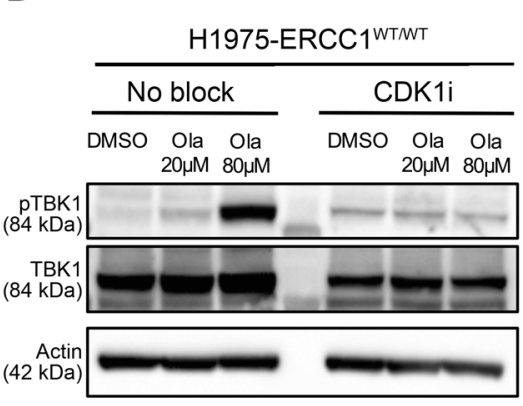

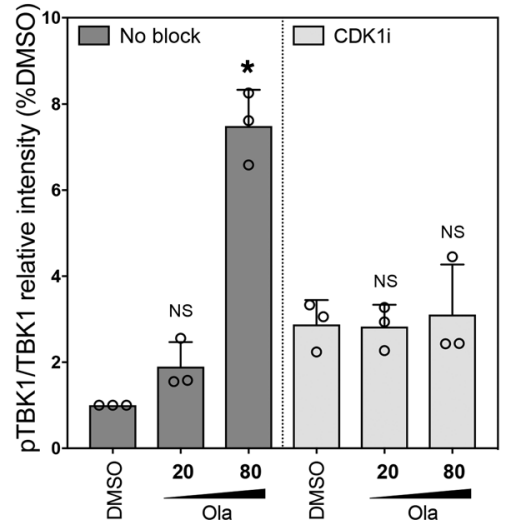

Figure 6. cGAS-mediated detection of PARPi-induced CCFs activates STING signaling in an ERCC1-dependent manner. (A and B) Western blot of pTBK1 in A549-ERCC1 ${ }^{\text {TT/WT }}$ and A549-ERCC1 ${ }^{-1-}$ cells (A) or H1975-ERCC1 ${ }^{\text {WT/WT }}$ and H1975-ERCC1 ${ }^{-/-}$cells (B) upon PARPi exposure. Cells were exposed for 48 hours to DMSO (vehicle) and a range of doses of Ola (A), or DMSO, $25 \mu \mathrm{M}$ Ruca, and $40 \mu \mathrm{M}$ Ola (B). Lysates were probed with the indicated antibodies. (C) Western blot of pTBK1 in DMSO- or Ruca-treated H1975-ERCC1WT/WT cells in the context of siRNA silencing of cGAS/STING. Cells were transfected with RNAiMAX (Thermo Fisher Scientific), siCTRL, siSTING, sicGAS, or siSTING + sicCAS and exposed to DMSO (vehicle) or $25 \mu \mathrm{M}$ Ruca, and lysates were probed with the indicated antibodies. (D) Western blot of PTBK1 in DMSO- or Ola-treated H1975-ERCC1 ${ }^{\text {WT/WT }}$ cells upon cell cycle blockade. Cells were exposed to DMSO or $20 \mu \mathrm{M}$ or $80 \mu \mathrm{M}$ Ola in the presence or absence of the cell cycle blocker CDK1i RO-3306. Lysates were probed with the indicated antibodies. Graph: pTBK1/TBK1 intensity was measured for each condition and normalized to DMSO. Mean $\pm \mathrm{SD}, n=3 ;{ }^{*} P<0.05$, Kruskal-Wallis test and post hoc Dunn's test, relative to DMSO control. 
A RT-qPCR A549-ERCC 1WTwt

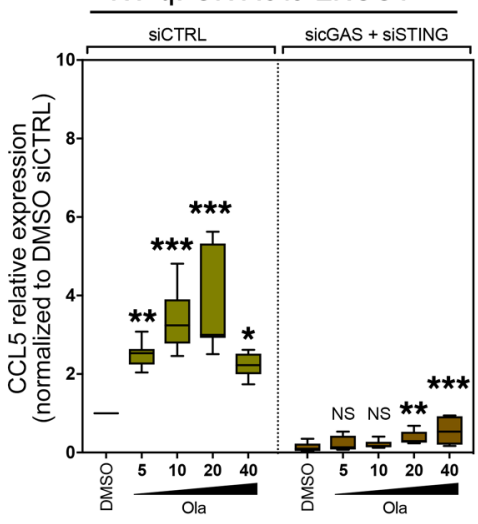

RT-qPCR A549-ERCC1

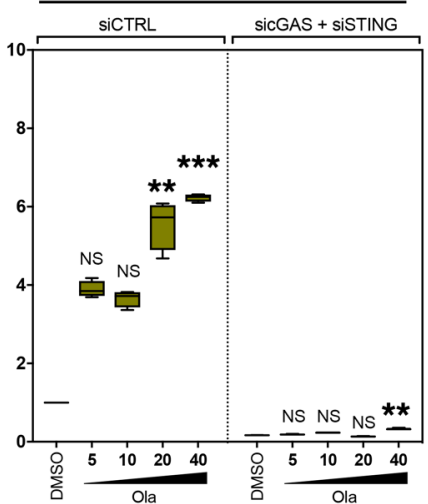

B RT-qPCR A549-ERCC1 isogenics

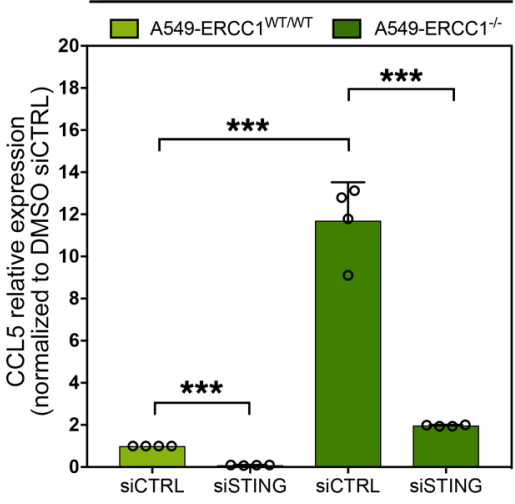

C

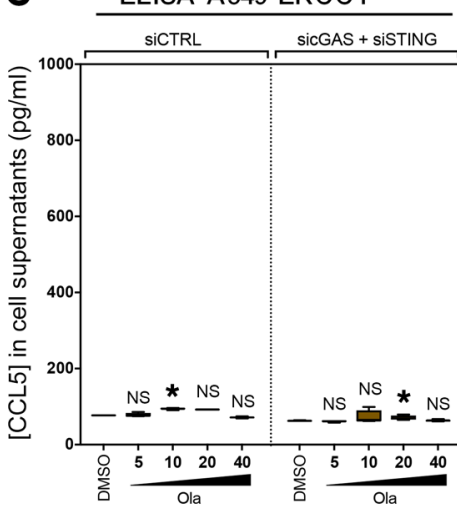

ELISA A549-ERCC $1^{+/}$-

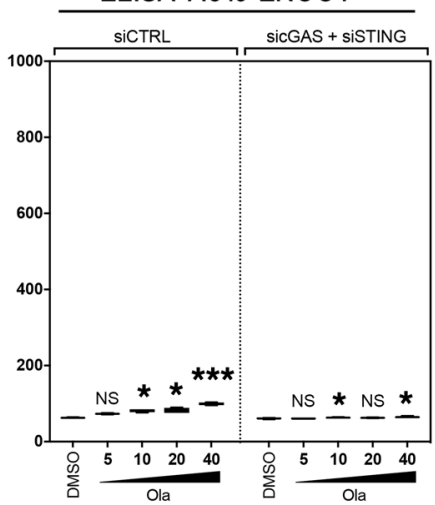

ELISA A549-ERCC 1

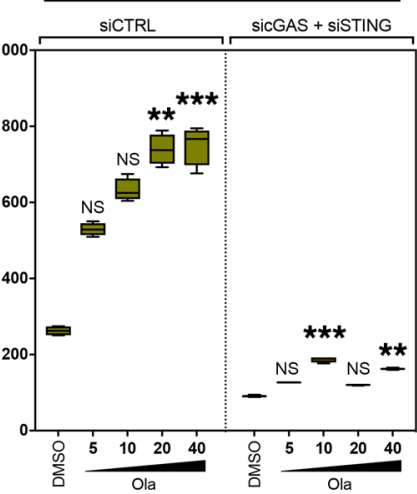

ELISA A549-ERCC1/ + isoform 202

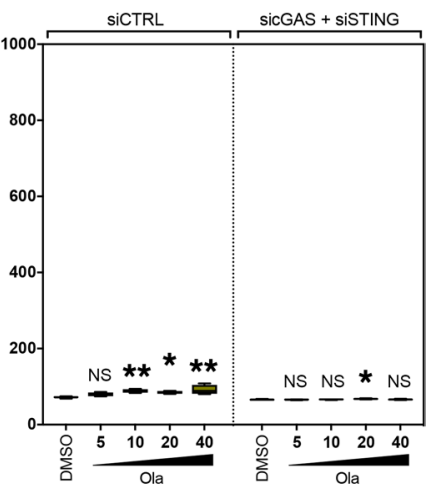

D A549-ERCC 1WTWT
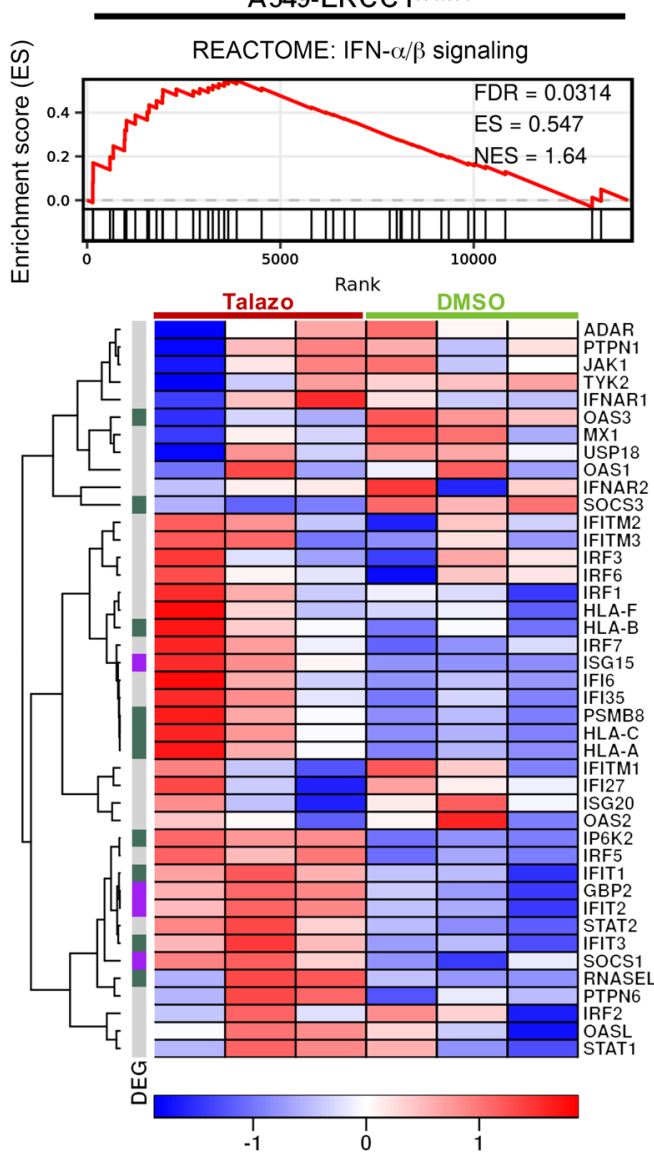

E A549-ERCC1
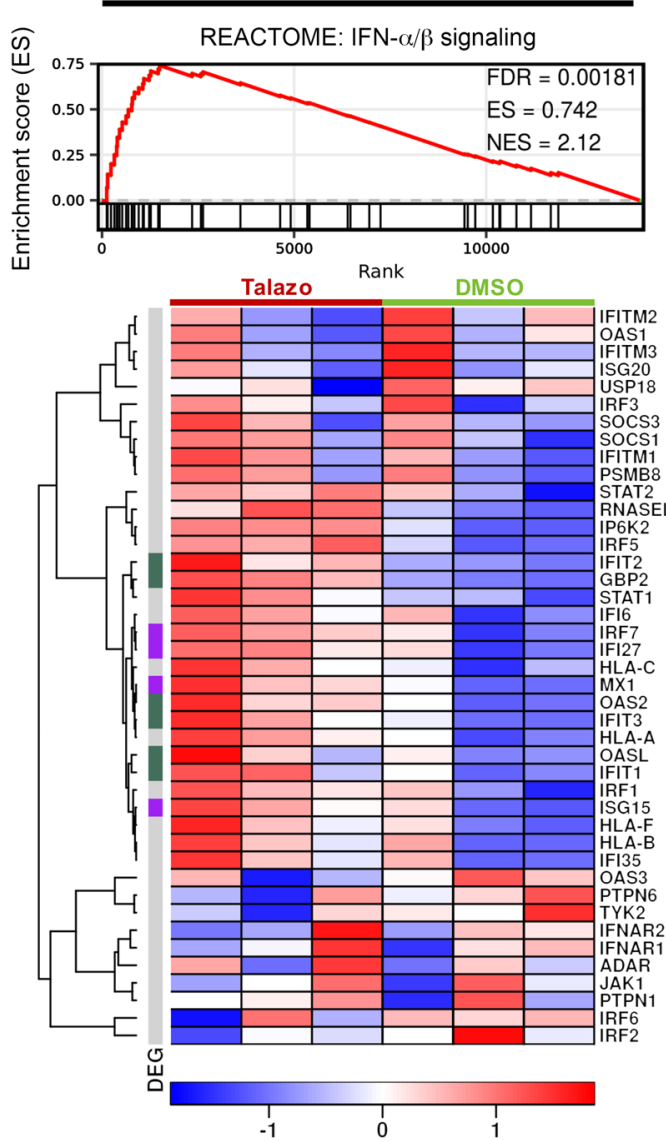
Figure 7. PARPi induce secretion of the chemotactic chemokine CCL5 in a cGAS/STING-dependent manner, and activate type I IFN signaling. (A) RT-qPCR analysis of RNA isolated from Ola-exposed A549-ERCC1 ${ }^{\text {WT/WT }}$ and A549-ERCC1/- cells, in the presence or absence of cGAS/STING silencing by siRNA. Cells were transfected with siCTRL or sicGAS + siSTING and treated for 72 hours with DMSO or a range of doses of Ola $(\mu \mathrm{M})$. CCL5 mRNAs were analyzed relative to CAPDH. Box-and-whisker plots show arbitrary units of gene expression, normalized to DMSO-treated control. Boxes indicate median and lower and upper quartiles; whiskers indicate the $5^{\text {th }}$ to $95^{\text {th }}$ percentile range; $n=12$, Kruskal-Wallis test and post hoc Dunn's test, relative to DMSO control. (B) RT-qPCR analysis of RNA isolated from A549-ERCC1 ${ }^{\text {WT/WT }}$ and A549-ERCC1 $1^{-1-}$ cells, in the presence or absence of CGAS/STING silencing by siRNA. Cells were transfected with siCTRL or sicCAS + siSTING. CCL5 mRNAs were analyzed relative to CAPDH. Shown are arbitrary units of gene expression, normalized to A549-ERCC1 ${ }^{\text {WT/WT }}$ DMSO-treated control. Mean $\pm S D, n=4$, 2-way ANOVA. (C) Quantitative analysis of CCL5 secretion in A549-ERCC1 isogenic cell supernatants upon Ola exposure, in the presence or absence of cGAS/STING silencing by siRNA. Cells were transfected with siCTRL or sicGAS + siSTING and treated for 72 hours with DMSO or a dose range of Ola $(\mu \mathrm{M})$. Supernatants were collected and analyzed by ELISA for detection of CCL5. Box-and-whisker plots show CCL5 concentrations. Boxes indicate median and lower and upper quartiles; whiskers indicate the 5th to 95th percentile range; $n=4$, Kruskal-Wallis test and post hoc Dunn's test, relative to DMSO control. ( $\mathbf{D}$ and $\mathbf{E}$ ) GSEA of the REACTOME pathway "IFN- $\alpha / \beta$ signaling" in talazoparib- (Talazo-) versus DMSO-treated A549-ERCC1 ${ }^{\text {WT/WT }}$ cells (D) or A549-ERCC1-/- cells (E). A heatmap showing the genes of the pathway is shown below. $n=3$; heatmap scale is a $Z$ score. Purple, significantly DEGs with FDR $<0.05$ and $|\mathrm{LFC}|>1$; green, significantly DEGs with $\mathrm{FDR}<0.05$ and $|\mathrm{LFC}|>0.58$; gray, nonsignificantly DEGs. ${ }^{*} P<0.05,{ }^{* *} P<0.01,{ }^{* * *} P<0.001$.

PARPi-induced CCFs activate cGAS/STING signaling in ERCC1-deficient NSCLC cells. To investigate the potential of PARPi-induced CCFs to activate cGAS/STING signaling, we assessed the ability of cGAS to form foci through relocalization to CCFs. Immunofluorescence staining of cGAS in A549-ERCC1 isogenic cell lines exposed to increasing concentrations of rucaparib or olaparib revealed a significant dose-dependent increase in the number of cytoplasmic cGAS foci in A549-ERCC1 ${ }^{-/}$and A549-ERCC1 ${ }^{+/-}$cells, but not in A549-ERCC1 ${ }^{\text {WT/WT }}$ cells (Figure 5, $A$ and $\mathrm{B} ; P=0.0122, P=0.0123$, and $P=0.1657$, respectively; Kruskal-Wallis test). Similar results were obtained in the independent H1975-ERCC1 isogenic NSCLC model (Supplemental Figure 4, $A$ and B). Increased formation of cytoplasmic cGAS foci was also detected in SUM149-BRCA1 ${ }_{\text {mut }}$ cells after PARPi exposure, at a higher level than in SUM149-BRCA1 ${ }_{\text {rev }}$ cells (1.4-fold difference at $20 \mu \mathrm{M}$ olaparib, $P=0.0033$, 2-way ANOVA, post hoc Šidák's test) (Figure 5, C and D). More importantly, no increase in cytoplasmic cGAS foci was detected in the PARPi-resistant SUM149-PARP1 ${ }^{-1-}$ cell line, supporting that the minimal levels of CCFs measured in this cell line did not trigger cGAS relocalization. Analysis of cGAS staining intensity within CCFs revealed a significant increase in cGAS foci/CCF colocalization upon PARPi exposure in both NSCLC ERCC1 isogenic models (Figure 5E and Supplemental Figure 4C; $P<0.0001$, Kruskal-Wallis test), thus confirming that cGAS relocalized to CCFs following PARPi exposure.

We then investigated whether the detection of CCFs by cGAS could activate STING signaling. Analysis of phosphorylated TBK1 (pTBK1, a key downstream effector of STING signaling) revealed a dose-dependent increase upon PARPi exposure in A549-ERCC1 ${ }^{-/-}$and A549-ERCC1 ${ }^{+/-}$models, but not A549-
ERCC1 ${ }^{\text {WT/WT }}$ cells (Figure 6A and Supplemental Figure 4D). Consistent results were observed in the H1975-ERCC1 isogenic model, where an increase in pTBK1 was detected upon PARPi exposure, which was more pronounced in the H1975-ERCC1 ${ }^{-1-}$ cells (Figure 6B and Supplemental Figure 4E). This suggested the ERCC1 dependence of PARPi-induced TBK1 phosphorylation. Furthermore, while silencing of either cGAS or STING only moderately decreased PARPi-induced TBK1 phosphorylation in A549-ERCC1 ${ }^{-/}$cells (Supplemental Figure 4F), simultaneous silencing of both sensors allowed complete abrogation of pTBK1, as observed in H1975-ERCC1 ${ }^{\mathrm{WT} / \mathrm{WT}}$ cells (Figure 6C). We then evaluated pTBK1 in the SUM149-BRCA1 isogenic TNBC model. Consistent with results obtained in the NSCLC models, we found a dose-dependent increase in pTBK1 upon PARPi exposure in SUM149-BRCA1 $1_{\text {mut }}$ but not SUM149-BRCA1 $1_{\text {rev }}$ cells (Supplemental Figure 4G). A corresponding increase in phosphorylation of several transcription factors involved in the final steps of the STING signaling cascade, including p65-NF-кB, IRF3, and IRF7, confirmed activation of the pathway in SUM149-BRCA1 ${ }_{\text {mut }}$ cells (Supplemental Figure 4G).

Because CCFs can also be detected by alternative STING-independent pattern recognition receptors (PRRs), namely the Tolllike receptor (TLR) and RIG-1-like receptor (RLR) pathways, we monitored the activation of those pathways after PARPi exposure. No activation of the TLR or RLR effectors could be detected upon PARPi exposure (Supplemental Figure 4, H and I), suggesting that the detection of CCFs following PARP inhibition is relatively specific to the cGAS/STING pathway.

Because our previous observations showed cell cycle dependence of CCF formation (Figure 4, E and F), we monitored TBK1 phosphorylation upon cell cycle blockade by CDK1i. Although CDK1i itself appeared to increase baseline pTBK1, we could detect an abrogation of the previously observed increase in TBK1 phosphorylation upon PARPi exposure (Figure 6D). Collectively, these results support the notion that PARPi-induced CCFs activate cGAS/STING signaling and that this effect relies on the presence of specific DNA repair defects.

PARPi promote secretion of CCL 5 and activate type I IFN signaling. Increased production of the chemotactic chemokine CCL5 has been previously reported in the context of DDR deficiency and following treatment with S phase-dependent DNA-damaging agents or ionizing radiation $(17,19,20)$. We therefore hypothesized that PARPi might enhance CCL5 production, especially in ERCC1-deficient cells. Quantitative RT-PCR (RT-qPCR) analyses of CCL5 mRNAs revealed dose-dependent activation of CCL5 transcription in both

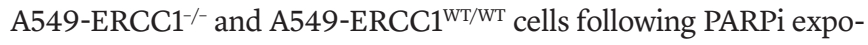
sure (Figure 7A), while baseline expression levels were 12-fold higher in A549-ERCC1 ${ }^{-/}$cells compared with A549-ERCC1 ${ }^{\text {WT/WT }}$ cells (Figure $7 \mathrm{~B}$ ). Consistent with these observations, quantification of CCL5 protein concentration in cell supernatants using ELISA revealed a similar dose-dependent increase in CCL5 secretion upon PARPi exposure in A549-ERCC1 ${ }^{-/}$but not A549-ERCC1 ${ }^{\text {WT/WT }}$, A549-ER$\mathrm{CC1}^{+-}$, or A549-ERCC1 ${ }^{-/-}+$isoform 202 cells (Figure 7C). Depletion of STING and cGAS by siRNA resulted in a suppression of CCL5 transcription and secretion, suggesting the involvement of cGAS/ STING in this process (Figure 7, A-C). Similarly, SUM149-BRCA1 ${ }_{\text {mut }}$ cells, but not SUM149-BRCA1 ${ }_{\text {rev }}$ cells, displayed enhanced expres- 
A
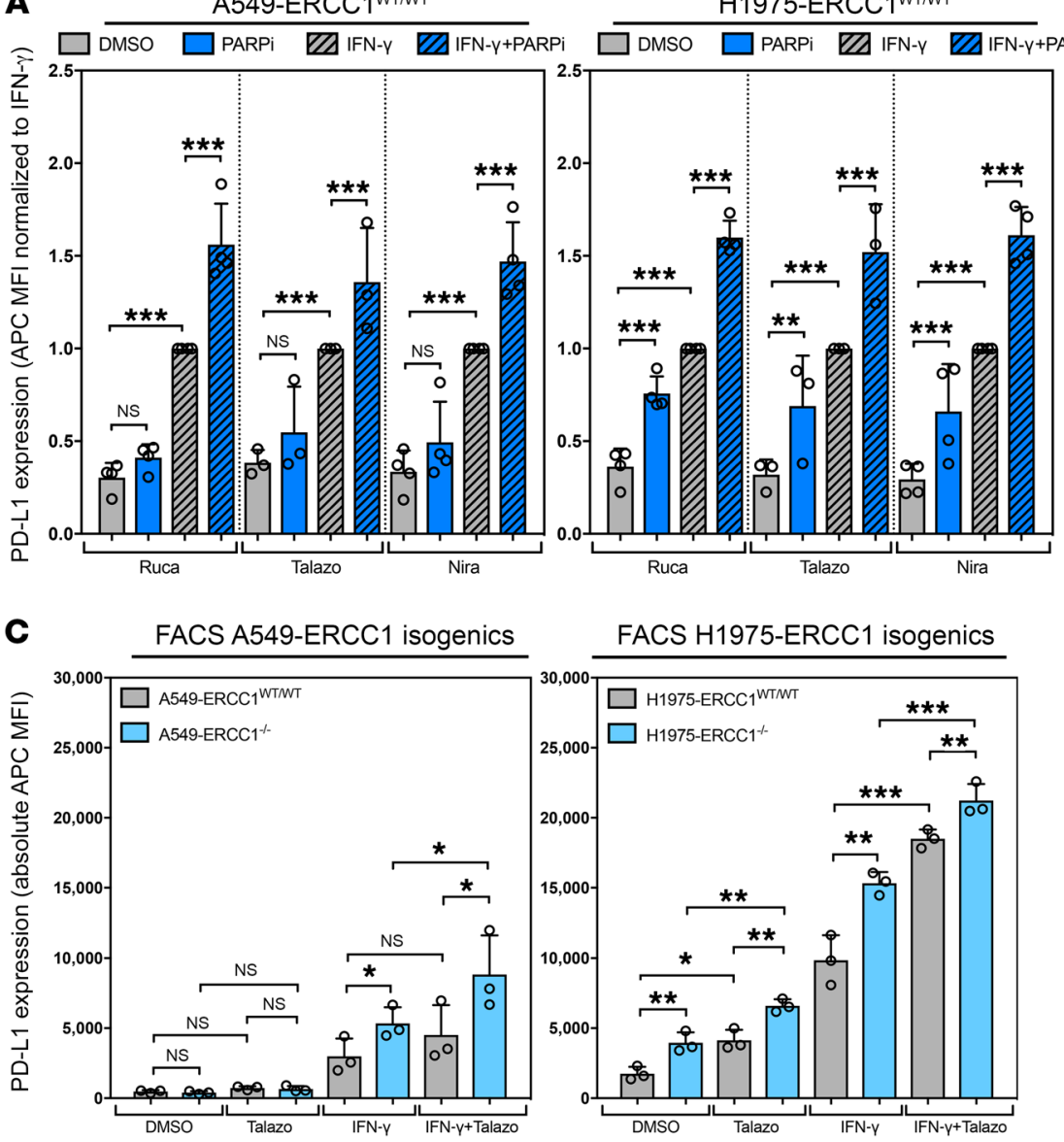

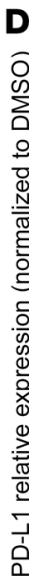
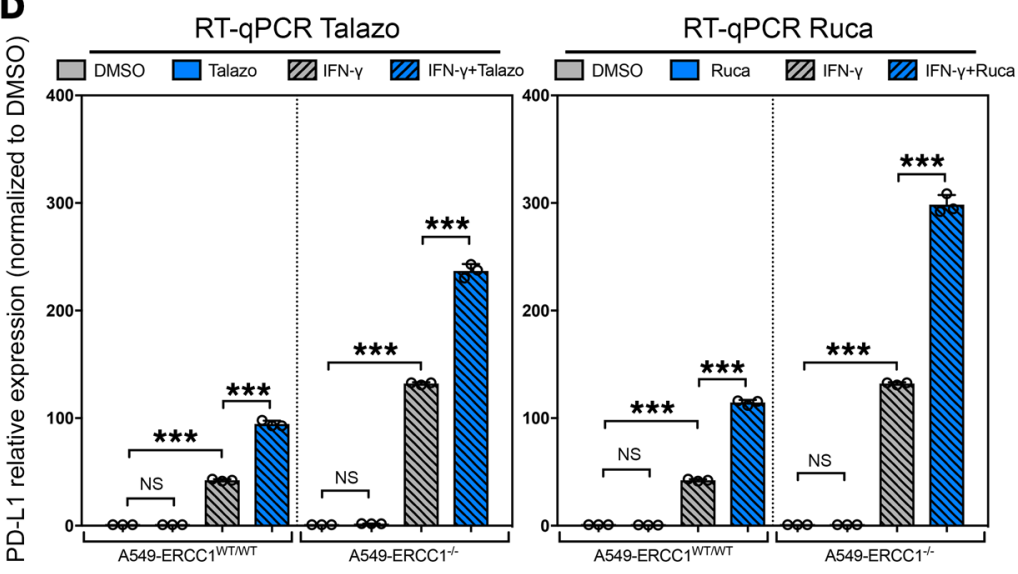

B
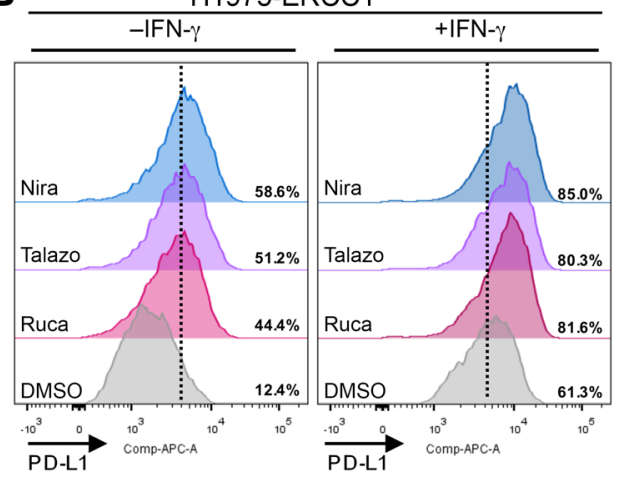
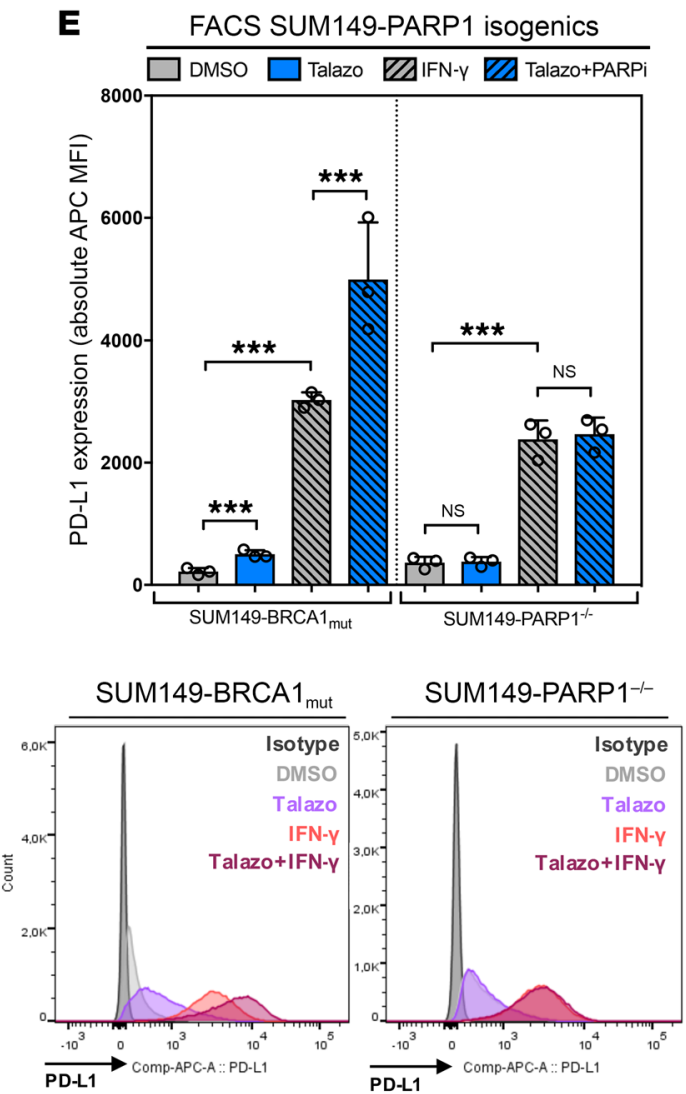

Figure 8. ERCC1 deficiency and PARPi exposure potentiate IFN- $\gamma$-induced cell-surface PD-L1 expression. (A) Quantification of PD-L1 cell surface expression by flow cytometry in H1975-ERCC1WT/WT and A549-ERCC1WT/WT cells upon PARPi and IFN- $\gamma$ exposure. Cells were treated for 48 hours with DMSO, 15 $\mu \mathrm{M}$ Ruca, $3 \mu \mathrm{M}$ Talazo, $10 \mu \mathrm{M}$ niraparib (Nira), and/or $500 \mathrm{U} / \mathrm{ml}$ IFN- $\gamma$. MFI \pm SD normalized to IFN- $\gamma ; n=4$, 2-way ANOVA and post hoc Tukey's test. (B) Corresponding flow cytometry histograms; shown is the percentage of PD-L1-positive cells. (C) Quantification of PD-L1 cell surface expression by flow cytometry in A549-ERCC1 ${ }^{\text {WT/WT }}$, A549-ERCC1 ${ }^{-1-}, \mathrm{H} 1975-E R C C 1^{\text {WT/WT }}$, and H1975-ERCC1 ${ }^{-/-}$cells treated for 48 hours with DMSO, $3 \mu \mathrm{M}$ Talazo, and/or $500 \mathrm{U} / \mathrm{ml}$ IFN- $\gamma$. MFI \pm SD, 2-way ANOVA and post hoc Tukey's test. (D) RT-qPCR analysis of RNA isolated from A549-ERCC1 ${ }^{\text {WT/WT }}$ and A549-ERCC1-1- cells exposed to PARPi and/or IFN- $\gamma$. Cells were treated for 48 hours with DMSO, $3 \mu \mathrm{M}$ Talazo, or $13.5 \mu \mathrm{M}$ Ruca, and/or $500 \mathrm{U} / \mathrm{ml}$ IFN- $\gamma$. PD-L1 mRNAs were analyzed relative to GAPDH (to control for CDNA quantity). Shown are arbitrary units of gene expression, normalized to A549-ERCC1 ${ }^{\text {WT/WT }}$ DMSO-treated control. Mean \pm SD, $n=3$, 2-way ANOVA and post hoc Tukey's test. (E) Quantification of PD-L1 cell surface expression by flow cytometry in SUM149-BRCA1 ${ }_{\text {mut }}$ and SUM149-PARP1 ${ }^{-1-}$ cells treated for 48 hours with DMSO, $3 \mu \mathrm{M}$ Talazo, and/or $500 \mathrm{U} / \mathrm{ml}$ IFN- $\gamma$. MFI \pm SD, $n=3$, 2-way ANOVA and post hoc Tukey's test. Corresponding flow cytometry histograms are shown at the bottom. ${ }^{*} P<0.05,{ }^{* *} P<0.01,{ }^{* * *} P<0.001$. 

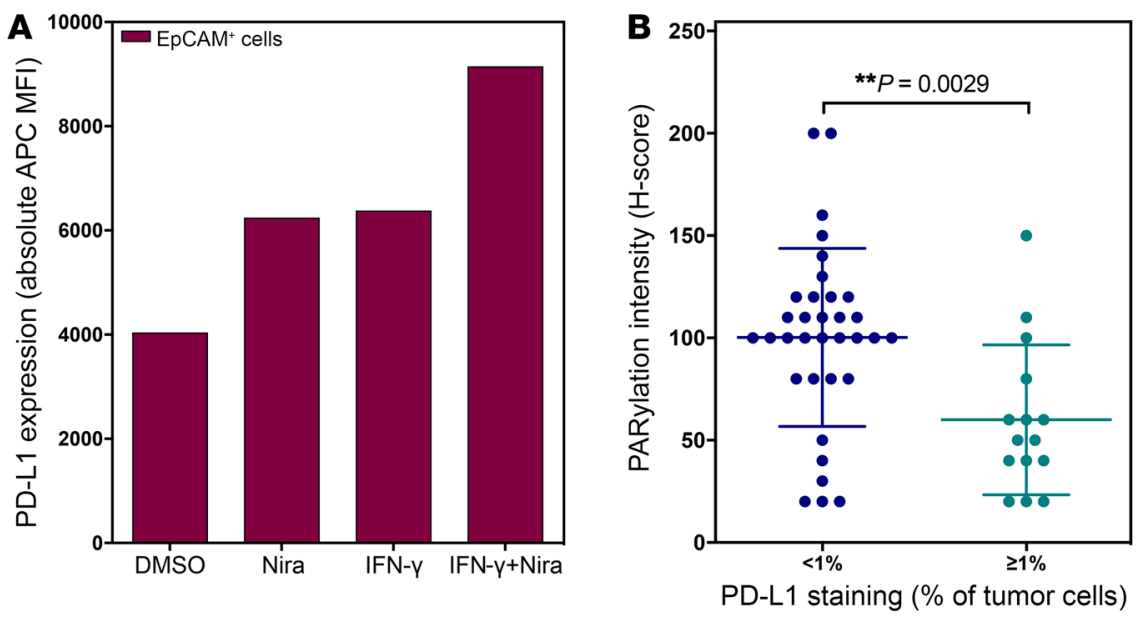

C

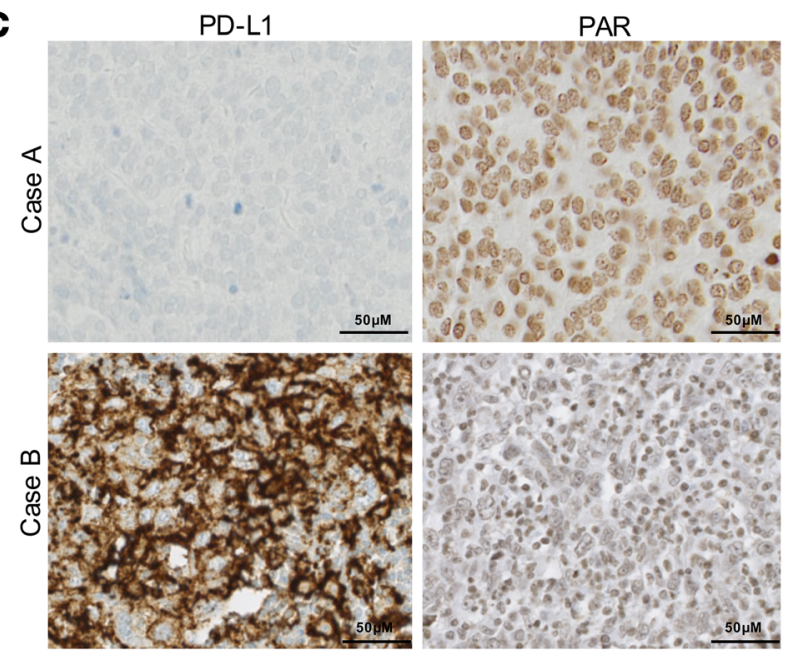

Figure 9. PARPi induce PD-L1 expression in patient-derived NSCLC cells and high PD-L1 expression associates with low PARylation in human NSCLC samples. (A) Quantification of PD-L1 cell surface expression by flow cytometry in the EpCAM-positive cells of a pleural effusion sample upon PARPi and/or IFN- $\gamma$ exposure. Cells were treated in vitro for 48 hours with DMSO or 10 $\mu \mathrm{M}$ Nira, $500 \mathrm{U} / \mathrm{ml}$ IFN $-\gamma$, or both. MFI of a single staining is shown. (B) Scatter box plot showing PARylation levels and tumor cell expression of PD-L1 (as assessed by IHC staining) in a series of resected stage I/II NSCLC (invasive adenocarcinomas and squamous cell carcinomas) samples ( $n$ = 49). Mann-Whitney $U$ test. (C) Representative images of PAR and PD-L1 IHC stainings in surgical specimens of NSCLC. Case A shows low PD-L1 staining in tumor cells and high PARylation levels case B shows high PD-L1 staining in tumor cells and low PARylation levels. Scale bars: $50 \mu \mathrm{m}$. sion and secretion of CCL5 following treatment with olaparib, which was reduced upon co-depletion of STING and cGAS (Supplemental Figure 5, A and B).

We next evaluated whether the production of other major chemokines was upregulated by PARPi exposure. Although a significant increase in IFNB1 transcription was observed in SUM149-BRCA1 $1_{\text {mut }}$ cells exposed to olaparib (Supplemental Figure $5 \mathrm{C}$ ), no increase in the corresponding secreted protein could be detected in cell supernatants (Supplemental Figure 5D). Similarly, no secretion of IFN- $\gamma$ or TNF- $\alpha$ was detected after olaparib exposure in this cell line (Supplemental Figure 5E).

To comprehensively characterize cell-autonomous immunomodulation induced by PARPi, we performed RNA-Seq on A549-ER$\mathrm{CC}^{\mathrm{WT} / \mathrm{WT}}$ and A549-ERCC1 ${ }^{-/-}$cells exposed to talazoparib, the most potent and specific clinical PARPi. This demonstrated significant upregulation of a number of genes involved in type I IFN signaling (Figure 7, D and E), which was associated with a significant enrichment of the type I IFN signature in A549-ERCC1-/ cells (NES = 2.12; $\mathrm{FDR}=0.0018$ ). Although this upregulation was observed in both A549-ERCC1 ${ }^{-/-}$and A549-ERCC1 ${ }^{\mathrm{WT} / \mathrm{WT}}$ cells, the corresponding enrichment was more modest in WT A549 cells (NES = 1.64; FDR $=0.0314$ ), highlighting the role of ERCC1 deficiency in potentiating this phenotype. These data support the hypothesis that PARPi specif- ically activate, in a cell-autonomous fashion in NSCLC cells, a type I IFN immune response that is enhanced in the context of ERCC1 deficiency, and can be linked to the secretion of CCL5 in our models.

PARPi synergize with IFN- $\gamma$ to induce cell-surface PD-L1 expression. PD-L1 is a major immune checkpoint protein expressed by tumor cells to evade immunosurveillance. PD-L1 induction is classically triggered by IFN- $\gamma$, which mediates the activation of type II IFN signaling cascade (38), but recent data suggest that platinum-based chemotherapies (39) as well as some PARPi (40) can also transiently enhance PD-L1 expression. Although the mechanisms underlying these effects are largely unknown, activation of PD-L1 expression following S phase-specific DNA damage has been associated with cGAS/STING recruitment (17). Because our previous data mostly pointed toward stimulation of type I IFN signaling through cGAS/STING in response to PARPi, we questioned whether PARPi could synergize with IFN- $\gamma$ to induce PD-L1 expression and whether ERCC1 status would influence this modulation. We exposed A549-ERCC1 isogenic and H1975-ERCC1 isogenic cell lines to PARPi and/or IFN- $\gamma$, and monitored cell-surface PD-L1 expression. Consistent with the published literature (40), we observed a significant dose-dependent upregulation of PD-L1 expression after treatment with rucaparib, talazoparib, or niraparib as monotherapy in both models (Figure 8, A and B, and 


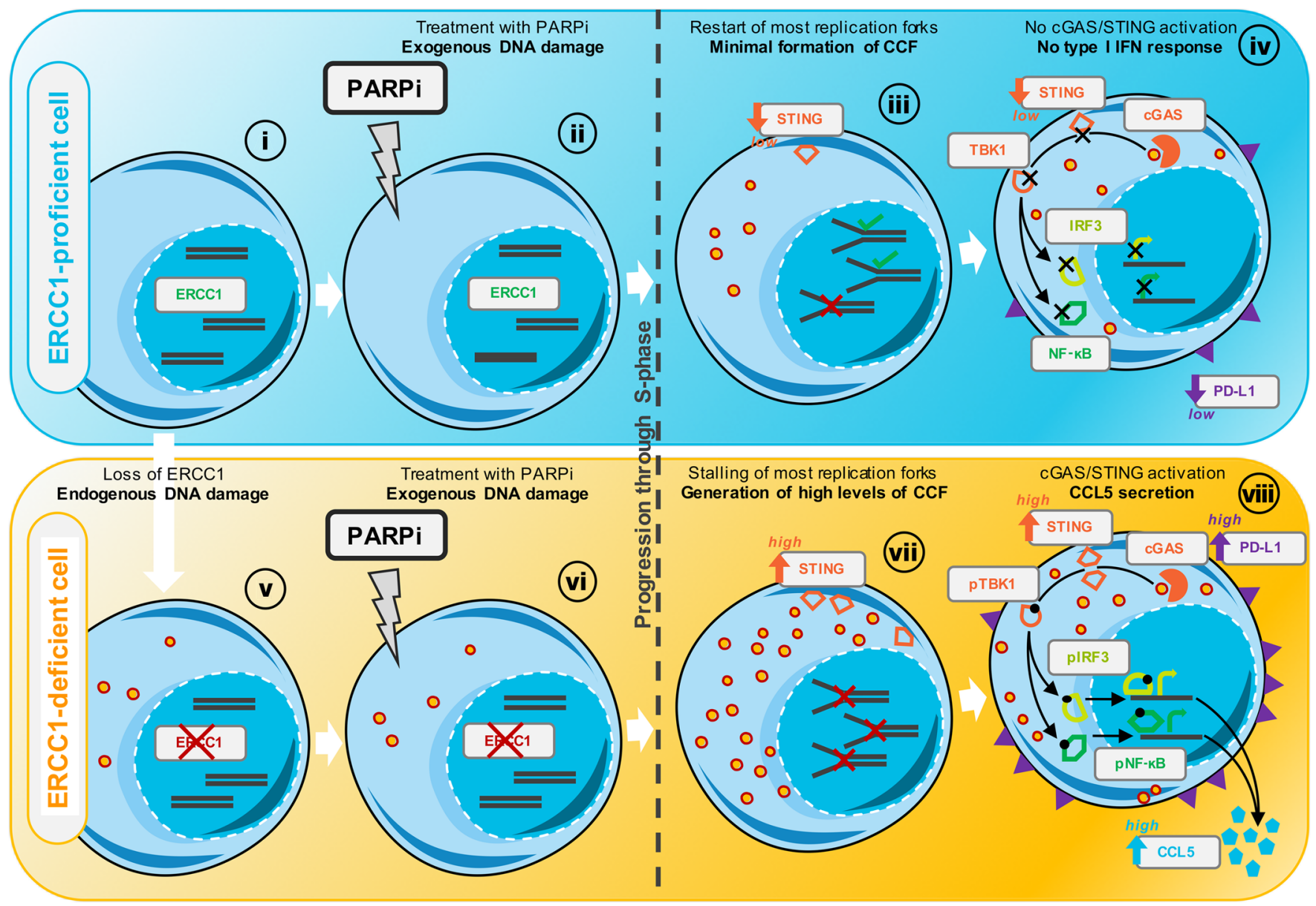

Figure 10. Model of ERCC1 defect-dependent activation of cGAS/STING following PARPi exposure. (i) ERCC1WT/WT cells have a functional DDR and adequately maintain genome integrity. (ii) Upon PARPi exposure, exogenous DNA damage is triggered, mostly initiated by PARP1 itself trapped onto the DNA at sites of spontaneous single-strand breaks (SSBs). (iii) During the $S$ phase of the cell cycle, trapped PARP1 generates lesions that prevent the progression of replication forks, leading to fork stalling and subsequent formation of DSBs. In ERCC1WT/WT cells, most trapped PARP1 lesions are removed, which enables the processing of DSBs through HR and eventually allows replication to restart. Residual inadequately repaired lesions cause moderate formation of CCFs. (iv) The low levels of CCFs generated are unable to trigger the pTBK1/IRF3/NF-kB signaling cascade or subsequent transcription of type I IFN genes; PD-L1 is moderately induced. (v) ERCC1 ${ }^{-/-}$cells are exposed to increased endogenous DNA damage following the loss of ERCC1. This generates minimal levels of CCFs. (vi) Upon PARPi exposure, ERCC1 $1^{-1-}$ cells are subjected to an additional exogenous source of DNA damage. (vii) During the $S$ phase of the cell cycle, trapped PARP1 generates lesions that prevent the progression of replication forks, leading to stalling of forks and subsequent formation of DSBs. In the absence of ERCC1, trapped PARP1 lesions cannot be adequately resolved, which triggers increased DSBs and eventually generates high levels of CCFs. (viii) CCFs are detected by cGAS and, due to the enhanced expression of STING in ERCC1 ${ }^{-1-}$ cells, these efficiently activate cGAS/STING signaling. Activated STING homodimer phosphorylates TBK1, which in turn phosphorylates IRF3 and NF-KB; this triggers their translocation into the nucleus and results in the transcription of type I IFN genes: CCL5 and other type I IFN cytokines are secreted. Higher PD-L1 expression is induced at the cell surface.

Supplemental Figure 6A). Interestingly, cotreatment with PARPi plus IFN- $\gamma$ was synergistic and could potentiate the induction of PD-L1 expression (Figure 8, A and B). This induction was more profound in A549-ERCC1 ${ }^{-/}$and $\mathrm{H} 1975-\mathrm{ERCC1}^{-/-}$models when compared with their ERCC1-proficient counterparts, at both the cell surface and transcriptional levels (Figure 8, C and D). To assess the specificity of the observed effect, we evaluated the membrane expression of TLR4, an immune-related cell surface marker that has been described to be positively correlated with PD-L1 expression in NSCLC samples (41). No TLR4 induction could be detected under similar conditions, suggesting that PD-L1 induction may be relatively specific (Supplemental Figure 6B). We finally confirmed that the observed PD-L1 induction resulted from an on-target effect of PARPi using the SUM149-PARP1 isogenic mod- el: although a significant synergy between talazoparib and IFN- $\gamma$ could be observed in parental SUM149-BRCA1 ${ }_{\text {mut }}$ cells, this effect was completely abrogated in SUM149-PARP1 ${ }^{-/-}$cells, where only IFN- $\gamma$ could induce cell-surface PD-L1 (Figure 8E).

To explore this interaction between PARPi and type II IFN signaling, we assessed whether PARPi could modulate the activation of STAT1, a key protein in the IFN- $\gamma$ signaling pathway that mediates PD-L1 induction. Although exposure to PARPi monotherapy did not induce STAT1 phosphorylation, enhanced STAT1 phosphorylation was detected following combination treatment of PARPi plus IFN- $\gamma$, compared with IFN- $\gamma$ alone (Supplemental Figure 6C). Interestingly, this effect was abrogated upon cell cycle blockade with the CDK1i RO-3306, suggesting that progression through the cell cycle is required for the potentiation of STAT signaling by PARPi. 
We next sought to assess whether these mechanisms would also operate in patients and exposed fresh cells from a patient's NSCLC pleural effusion sample to PARPi and IFN- $\gamma$. After selection of epithelial cell adhesion molecule-positive $\left(\mathrm{EpCAM}^{+}\right)$ cells, we quantified PD-L1 cell-surface expression by flow cytometry. This revealed a similar PD-L1 induction upon PARPi exposure, which was again potentiated by IFN- $\gamma$, consistent with our in vitro findings (Figure 9A).

As PARPi have a dual mechanism of action (i.e., inhibition of the PARylation catalytic activity of PARP1 and PARP1 trapping), we also sought to evaluate whether PARylation levels or PARP1 expression would correlate with PD-L1 expression in human tumor samples. We performed immunostaining of PAR, PARP1, and PD-L1 in a series of 49 resected stage I/II NSCLC samples. No correlation between PARP1 expression and PD-L1 staining (Supplemental Figure 6, E and F) was found. A significant inverse correlation was found between PAR and PD-L1 levels on tumor cells (mean PARylation $\mathrm{H}$-score of 100 in PD-L1-low vs. 60 in PD-L1-high tumor cells; $P=0.003$; Mann-Whitney $U$ test; Figure 9, B and C) but not PD-L1 expression on immune cells (Supplemental Figure 6D), consistent with a previous report in breast cancer specimens (40).

A proposed model to explain cGAS/STING activation following PARPi exposure in tumor cells with ERCC1 deficiency. Although several scenarios may explain our findings, we propose the following model, which is consistent with our observations (Figure 10): $\mathrm{ERCC} 1^{\mathrm{WT} / \mathrm{WT}}$ cells adequately repair endogenous DNA lesions. PARPi exposure causes DNA damage, mostly initiated by PARP1 itself trapped onto the DNA at sites of spontaneous single-strand breaks. Trapped PARP1 generates stalled replication forks and subsequent DSBs during DNA replication. In ERCC1 $1^{\mathrm{WT} / \mathrm{WT}}$ cells, trapped PARP1 lesions can be adequately excised, a process in which ERCC1 is thought to play a major role (28). Following excision, repair of DSBs occurs through HR, and replication restarts. Residual unrepaired lesions cause minimal formation of CCFs, which are insufficient to trigger cGAS/STING signaling (Figure 10, i-iv). By contrast, ERCC1 ${ }^{-/-}$cells are exposed to increased levels of endogenous DNA damage, which are further enhanced upon PARPi exposure. Trapped-PARP1 lesions cannot be appropriately resolved in the absence of ERCC1 (28), which prevents the subsequent processing of DSBs through HR (Figure 10, v-vii). This results in the accumulation of stalled replication forks, subsequent DSBs, and unrepaired DNA lesions, which eventually causes increased micronuclei formation and CCF generation (Figure 10, vii). These are detected by cGAS, which activates the STING/ pTBK1 cascade and results in the transcription of type I IFN genes, including CCL5 (Figure 10, viii).

\section{Discussion}

DNA repair deficiency is a hallmark of cancer that has been therapeutically exploited for more than 50 years using DNA-damaging cytotoxic chemotherapies and more recently, with the advent of targeted DNA repair inhibitors such as PARPi, using synthetic lethality. Here, we show that multiple clinical PARPi can trigger the activation of innate immune pathways in a cell-autonomous fashion in vitro. By studying multiple isogenic models of DDR deficiency or PARPi resistance, and using fresh and archived tumor samples, we found that PARPi can elicit a cGAS/STING/
pTBK1/pIRF3/type I IFN response in cancer cells. Importantly, this response was exacerbated in ERCC1-deficient NSCLC contexts. We further showed that ERCC1 deficiency and PARPi were synergistic with IFN- $\gamma$ in inducing PD-L1 expression (Figures 8 and 9), thereby providing a rationale for strategies combining PARPi and anti-PD-(L) 1 therapies in appropriately molecularly selected populations.

Despite significant improvements in outcome brought by the advent of anti-PD-(L)1 therapies, NSCLC still represents the leading cause of cancer-related death. Only a minority of patients currently benefit from ICI, and strategies to turn "cold" tumors into "hot" tumors are being actively investigated. Interestingly, high TIL levels correlate with improved survival following neoadjuvant therapy in TNBC (42) - an observation that was also made in some NSCLC series in the adjuvant setting (43). In line with our findings, preclinical data evaluating rucaparib in combination with anti-PD-(L) 1 in syngeneic models of ovarian cancer have suggested a possible role for PARPi in promoting tumor lymphocytic infiltration, and increased benefit compared with anti-PD-(L)1 monotherapies in BRCA1-mutated tumors (44). Our observation that PARPi trigger cGAS/STING signaling (Figures 5 and 6) and favor secretion of lymphoattractant chemokines such as CCL5 in ERCC1-deficient NSCLC (Figure 7) suggests that PARPi could be used as immunomodulatory agents to trigger lymphocytic infiltration in this histotype. Conversely, adding anti-PD-(L)1 therapies to PARPi might overcome the immune escape mechanisms resulting from PARPi-induced PD-L1 upregulation on tumor cells. Importantly, recent data from the large randomized double-bind phase III study Keynote 189 (NCT02578680) evaluating the combination of pembrolizumab and platinum-based chemotherapy in first-line metastatic NSCLC have reported impressive benefits in progression-free and overall survival (HR for death $=0.49$; 95\% CI, 0.38-0.64; $P<0.001)(1)$. These results changed practice, setting up the framework for recommending anti-PD-(L)1 therapy in combination with platinum-based doublet chemotherapy in first-line advanced NSCLC. Interestingly and contrary to previous studies evaluating anti-PD-(L)1 monotherapy, this benefit was observed across all subgroups and was independent of baseline PD-L1 tumor expression score, suggesting that other tumor characteristics - such as DDR status - might play a role in response to this combination. Platinum sensitivity is a relevant phenotypical biomarker of sensitivity to PARPi (27), which suggests that PARPi - which are much better tolerated and do not cause severe bone marrow toxicity - might represent an interesting alternative or complement (in the maintenance setting) to platinum-based chemotherapy in combination with anti-PD-(L)1 agents in NSCLC. The development of clinical trials addressing this question is underway.

TMB is an important determinant of response to ICI (9, 10). While various DDR defects have been shown to critically contribute to the accumulation of mutations in the tumor genome, only some of them have proven their predictive value as biomarkers of response to anti-PD-(L) 1 therapy $(6,45)$. ERCC1 deficiency has been associated with increased mutation frequency and genomic instability in mice (46), an observation that is consistent with the known roles of ERCC1 in processes that ensure chromosomal stability and maintenance of genome 
integrity, such as the interstrand crosslink DNA repair pathway (47) and resolution of Holliday junctions (48). However, no formal studies have yet been conducted to assess whether ERCC1 status might influence TMB in NSCLC. This would be best addressed by interrogating large datasets (e.g., The Cancer Genome Atlas [TCGA]) for an inverse correlation between ERCC1 isoform 202 expression and TMB. Furthermore, whether ERCC1 status itself represents an independent predictive biomarker of response to anti-PD-(L) 1 in NSCLC is currently unknown and deserves further investigation.

Although our study focused on ERCC1 - the most frequent DDR deficiency in NSCLC - we can hypothesize that our findings may be applicable to other DDR defects detected at a lower frequency in NSCLC. For example, BRCA1, BRCA2, ATM, and MSH2 (mutated in 5\%, 6\%, 9\%, and 3\% of NSCLC cases, respectively; ref. 49) have also been associated with PARPi sensitivity and type I IFN signature (50-53). Interestingly, Teo et al. recently reported that somatic DDR alterations that were associated with improved clinical outcomes in platinum-treated patients with advanced urothelial carcinoma (54) also correlated with longer progression-free survival and overall survival upon anti-PD-(L)1 therapy (15). The authors reported that $25 \%$ of patients presented tumors with alterations in DDR genes, the most commonly altered genes being ATM, POLE, BRCA2, ERCC2, FANCA, and MSH6. Overall, these observations suggest that the interplay among DDR deficiency, platinum or PARPi sensitivity, and anticancer immune response operates in several tumor types and can involve multiple DDR defects.

cGAS is a potent sensor of almost any form of cytoplasmic DNA. It can detect free DNA, arising from reverse transcription of endogenous retroviruses (55) or mitochondrial DNA instability (56); and micronuclei, which arise from genomic instability and display chromatin-like structures, sometimes surrounded by a fragile lamin B1 membrane (19). Our data provide evidence that clinical PARPi can induce cytosolic DNA (Figures 3 and 4), which is at least in part in the form of micronuclei, and subsequently activates cGAS/STING signaling (Figures 5 and 6). In particular, our observation that these effects are abrogated in PARP1-null cells supports a direct on-target effect of these drugs, which is mostly mediated by their PARP-trapping potential (36). Furthermore, use of several specific isogenic models demonstrated that this effect is enhanced in several DNA repairdeficient contexts, notably ERCC1 and BRCA1 deficiency. This extends and is consistent with previous publications that reported activation of cGAS/STING by micronuclei following either ionizing radiation $(19,20)$ or $S$ phase-specific chemotherapies $(17,18)$, and published data that showed PARPi-mediated induction of IFN signals (notably increased STAT1 phosphorylation) in BRCA1-deficient contexts (20). Importantly, our observation that the immune phenotype of the ERCC1-heterozygous model closely resembles that of the $E R C C 1^{-/-}$clones suggests that our findings may be clinically relevant for NSCLC, where some residual expression of ERCC1 is usually retained.

In conclusion, we provide a scientific rationale for assessing PARPi in combination with anti-PD-(L)1 therapy in molecularly selected DNA repair-deficient populations. While several clinical trials are currently combining PARPi with anti-PD-(L) 1 in
BRCA1/2-mutant breast or ovarian cancers, other histologies, such as NSCLC in which anti-PD-(L) 1 therapies have already shown impressive results, might also benefit from such association. Basket clinical trials are planned to be launched soon to explore this question.

\section{Methods}

Additional methods are described in Supplemental Methods.

Study design. The aim of this study was to assess the immunomodulatory potential of PARPi, their ability to trigger specific cancer cell-associated immune responses, and their potential to enhance cancer cell immunogenicity through cell-autonomous mechanisms. Our prespecified hypothesis was that PARPi might have intrinsic immunomodulatory properties capable of activating immune signaling in a cell-autonomous fashion in cancer cells, specifically in those harboring DDR defects. Hence, we used a combination of in vitro isogenic systems of DDR-deficient and PARPi-sensitive or -resistant NSCLC and TNBC. All laboratory experiments were performed in at least 3 independent biological replicates to allow appropriate statistical calculations, using multiple well-characterized models and several PARPi evaluated in dose response experiments at clinically relevant concentrations. RNA-Seq was performed to generate at least 20 million reads per sample. For each condition, 3 biological replicates were included in the RNA-Seq analysis. Clinically relevant findings were further assessed in retrospectively analyzed archived patient tumor samples as well as one fresh prospectively collected pleural effusion.

Cell lines. SUM149, A549, and H1975 cells were obtained from ATCC. The generation of SUM149-BRCA1 $1_{\text {rev }}$ and SUM149$\mathrm{PARP}^{-/-}$secondary mutant cell lines was performed using CRISPR/ Cas 9 site-directed mutagenesis, as previously described $(35,36)$. The secondary mutant cell lines A549-ERCC1 ${ }^{-/-}$and H1975-ER$\mathrm{CC}^{-/}$were generated using zinc finger nuclease gene targeting, as described previously (31). SUM149 cells were cultured in Ham's F12 nutrient mixture with $10 \% \mathrm{FBS}, 1 \mu \mathrm{g} / \mathrm{ml}$ insulin, and $500 \mathrm{ng} /$ ml hydrocortisone. A549 and H1975 cells were cultured, respectively, with high-glucose DMEM and RPMI 1640 medium with $10 \%$ FBS. The cells were cultured under physiological conditions at $37^{\circ} \mathrm{C}$ and $5 \% \mathrm{CO}_{2}$. All cell lines were short tandem repeat (STR) typed using StemElite ID (Promega) to confirm identity prior to the study, and verified for mycoplasma contamination every 2 months using MycoAlert (Lonza).

Drugs and chemicals. The PARPi olaparib (AZD-2281, Astra Zeneca), rucaparib (PF-01367338, Clovis Oncology), and talazoparib (BMN-673, Pfizer) were purchased from Selleck Chemicals. Niraparib (MK-4827, Tesaro) was obtained from MedChemExpress. Inhibitor stock solutions were prepared in DMSO and stored in aliquots at $-80^{\circ} \mathrm{C}$. Hydroxyurea (HU), 5 -fluorouracil (5-FU), and the CDK1 inhibitor RO-3306 were obtained from Sigma-Aldrich. IFN- $\gamma$-1b (IMUKIN) was purchased from Boehringer.

Immunofluorescence and image analysis. Immunofluorescence assays were performed in 96-well plates. Cells were fixed in $4 \%$ paraformaldehyde (PFA) in PBS during 20 minutes at room temperature (RT), washed twice with PBS, and permeabilized with $0.5 \%$ Triton $\mathrm{X}-100$ in PBS for 10 minutes. After 2 additional washes, cells were blocked with 2\% BSA, 2\% FBS in PBS (IFF) for 1 hour at RT. Cells were then incubated with primary antibodies in IFF at $4^{\circ} \mathrm{C}$ overnight. 
The cells were then washed 3 times with PBS, each for 10 minutes, followed by incubation with Alexa Fluor 594-conjugated rabbit and Alexa Fluor 647-conjugated mouse secondary antibodies (Thermo Fisher Scientific), $1 \mu \mathrm{g} / \mathrm{ml}$ DAPI, and 1:400 PicoGreen (Thermo Fisher Scientific) in IFF for 1 hour at RT. After that, cells were washed again 3 times with PBS, and $100 \mu$ PBS was finally added to each well prior to imaging. Plates were imaged using an Operetta high-content imaging system (PerkinElmer). Quantification of the number of CCFs, micronuclei, and cGAS foci was performed under identical microscopy settings between samples, using the Columbus image analysis system (PerkinElmer). Twenty-five randomly selected fields containing more than 200 cells were analyzed within 3 individual replicates for each sample.

Detection of secreted cytokines. Detection of secreted cytokines in cell supernatants was performed through ELISA detection. Culture media were collected after a 72-hour culture, and cell numbers were counted for normalization. The media were dispensed in 96-well V-bottomed plates and centrifuged at $500 \mathrm{~g}$ for 5 minutes to pellet cells and debris. The resulting supernatants were used for ELISA detection with the following kits: Human CCL5 ELISA MAX Deluxe kit (catalog 440806), Human IFN- $\gamma$ ELISA MAX Standard kit (catalog 430103), and Human TNF- $\alpha$ ELISA MAX Deluxe kit (catalog 430206) from BioLegend; and Human IFN- $\beta$ ELISA kit (catalog 41410-1) from PBL Assay Science. Assays were performed in 4 replicates following the manufacturers' protocols. Absorbance was evaluated using a VICTOR multilabel plate reader (PerkinElmer).

Flow cytometry analyses. Cells were detached using Versene solution (Thermo Fisher Scientific) and transferred to round-bottom FACS tubes. After 5 minutes of centrifugation at $500 \mathrm{~g}$, the cells were washed with PBS and incubated at $4^{\circ} \mathrm{C}$ for 30 minutes with primary antibodies, diluted in 5\% BSA in PBS. The supernatants were removed after centrifugation, and the cells were washed again twice with PBS. The pellets were finally diluted in $250 \mu \mathrm{l} \mathrm{PBS}$ containing $1 \mu \mathrm{g} / \mathrm{ml}$ propidium iodide (PI) prior to analysis. Cell-surface expression of PD-L1 and TLR-4 was quantified and analyzed by flow cytometry on an LSR II flow cytometer (BD Biosciences). PI was used as a viability marker. Data analysis was performed using the FlowJo software package.
Statistics. No statistical methods were used to predetermine sample size. The experiments were not randomized. The investigators were not blinded to allocation during experiments and outcome assessment. All bar graphs show mean values with error bars (SD); 95\% confidence intervals were used, and significance was considered when $P$ was less than $0.05 ;{ }^{*} P<0.05,{ }^{* *} P<0.01,{ }^{* * *} P<0.001,{ }^{* * * *} P<0.0001$.

Study approval. No specific approval was required for this study, as no experiments were conducted in animals or humans. Informed consent was obtained from patients for the collection and study of pleural effusion samples.

Data availability. All RNA-Seq data sets generated as part of this study are publicly available at the European Nucleotide Archive (https://www.ebi.ac.uk/ena), under accession number PRJEB30090.

\section{Author contributions}

RMC, CJL, and SPV conceived and designed the study. RMC, GM, DBK, JA, ANJT, AA, SJP, SH, AM, JCS, CJL, and SPV developed the methodology. RMC, DBK, JA, SJP, JCS, CJL, and SPV acquired data. RMC, GM, JA, SH, CJL, SPV, and AL analyzed and interpreted data (including statistical analysis, biostatistics, and computational analysis). All authors wrote, reviewed, and/or revised the manuscript. RMC, GM, DBK, JA, MG, SJP, SH, AM, JCS, CJL, SPV, DM, CH, ND, MR, RM, IB, MLC, AK, and BB provided administrative, technical, or material support (e.g., reporting or organizing data, constructing databases). SPV, CJL, JCS, and AM supervised the study.

\section{Acknowledgments}

This work was funded by program grants to SPV from Integrated Cancer Research Site (SIRIC) SOCRATE-2 INCa-DGOSINSERM_12551 and ATIP-Avenir; program grants to CJL from Cancer Research UK and Breast Cancer Now, as well as a project grant to JCS from SIRIC-SOCRATE. RMC received funding from Fondation Philanthropia - Lombard Odier.

Address correspondence to: Sophie Postel-Vinay, DITEP, Gustave Roussy -114, Rue Edouard Vaillant, 94805 Villejuif, France. Phone: 33.01.42.11.43.43; Email: sophie.postel-vinay@gustaveroussy.fr.
1. Gandhi L, et al. Pembrolizumab plus chemotherapy in metastatic non-small-cell lung cancer. N Engl J Med. 2018;378(22):2078-2092.

2. Hellmann MD, et al. Nivolumab plus ipilimumab in lung cancer with a high tumor mutational burden. NEngl J Med. 2018;378(22):2093-2104.

3. Gettinger S, et al. Five-year follow-up of nivolum$\mathrm{ab}$ in previously treated advanced non-small-cell lung cancer: results from the CA209-003 Study. JClin Oncol. 2018;36(17):1675-1684.

4. Fruh M, Peters S. Genomic features of response to combination immunotherapy in lung cancer. Cancer Cell. 2018;33(5):791-793.

5. Chabanon RM, Pedrero M, Lefebvre C, Marabelle A, Soria JC, Postel-Vinay S. Mutational landscape and sensitivity to immune checkpoint blockers. Clin Cancer Res. 2016;22(17):4309-4321.

6. Le DT, et al. PD-1 Blockade in tumors with mismatch-repair deficiency. $N$ Engl JMed. 2015;372(26):2509-2520.

7. Mehnert JM, et al. Immune activation and response to pembrolizumab in POLE-mu- tant endometrial cancer. J Clin Invest. 2016;126(6):2334-2340.

8. Johanns TM, et al. Immunogenomics of hypermutated glioblastoma: a patient with germline POLE deficiency treated with checkpoint blockade immunotherapy. Cancer Discov. 2016;6(11):1230-1236.

9. Snyder A, et al. Genetic basis for clinical response to CTLA-4 blockade in melanoma. N Engl JMed. 2014;371(23):2189-2199.

10. Rizvi NA, et al. Cancer immunology. Mutational landscape determines sensitivity to PD-1 blockade in non-small cell lung cancer. Science. 2015;348(6230):124-128.

11. Hellmann MD, et al. Genomic features of response to combination immunotherapy in patients with advanced non-small-cell lung cancer. Cancer Cell. 2018;33(5):843-852.e4.

12. Hugo W, et al. Genomic and transcriptomic features of response to anti-pd-1 therapy in metastatic melanoma. Cell. 2016;165(1):35-44.

13. McGranahan N, et al. Clonal neoantigens elicit T cell immunoreactivity and sensitivity to immune checkpoint blockade. Science. 2016;351(6280):1463-1469.

14. Spranger S, et al. Density of immunogenic antigens does not explain the presence or absence of the T-cell-inflamed tumor microenvironment in melanoma. Proc Natl Acad Sci U S A 2016;113(48):E7759-E7E68.

15. Teo MY, et al. Alterations in DNA damage response and repair genes as potential marker of clinical benefit from PD-1/PD-L1 blockade in advanced urothelial cancers. J Clin Oncol. 2018;36(17):1685-1694.

16. Chen Q, Sun L, Chen ZJ. Regulation and function of the cGAS-STING pathway of cytosolic DNA sensing. Nat Immunol. 2016;17(10):1142-1149.

17. Parkes EE, et al. Activation of STING-dependent innate immune signaling by S-phase-specific DNA damage in breast cancer. J Natl Cancer Inst. 2017;109(1):djw199.

18. Erdal E, Haider S, Rehwinkel J, Harris AL, McHugh PJ. A prosurvival DNA damage-induced 
cytoplasmic interferon response is mediated by end resection factors and is limited by Trex1. Genes Dev. 2017;31(4):353-369.

19. Mackenzie KJ, et al. cGAS surveillance of micronuclei links genome instability to innate immunity. Nature. 2017;548(7668):461-465.

20. Harding SM, Benci JL, Irianto J, Discher DE, Minn AJ, Greenberg RA. Mitotic progression following DNA damage enables pattern recognition within micronuclei. Nature. 2017;548(7668):466-470.

21. Wang $\mathrm{H}$, et al. cGAS is essential for the antitumor effect of immune checkpoint blockade. Proc Natl Acad Sci USA. 2017;114(7):1637-1642.

22. Mullard A. Can innate immune system targets turn up the heat on 'cold' tumours? Nat Rev Drug Discov. 2018;17(1):3-5.

23. Postel-Vinay S, Vanhecke E, Olaussen KA, Lord CJ, Ashworth A, Soria JC. The potential of exploiting DNA-repair defects for optimizing lung cancer treatment. Nat Rev Clin Oncol. 2012;9(3):144-155.

24. Turner NC, et al. BRCA1 dysfunction in sporadic basal-like breast cancer. Oncogene. 2007;26(14):2126-2132.

25. Xu Y, et al. Promoter methylation of BRCA1 in triple-negative breast cancer predicts sensitivity to adjuvant chemotherapy. Ann Oncol. 2013;24(6):1498-1505.

26. Olaussen KA, et al. DNA repair by ERCC1 in non-small-cell lung cancer and cisplatin-based adjuvant chemotherapy. $N$ Engl J Med. 2006;355(10):983-991.

27. Tutt A, et al. Carboplatin in BRCA1/2-mutated and triple-negative breast cancer BRCAness subgroups: the TNT Trial. Nat Med.2018;24(5):628-637.

28. Postel-Vinay S, et al. A high-throughput screen identifies PARP1/ 2 inhibitors as a potential therapy for ERCC1-deficient non-small cell lung cancer. Oncogene. 2013;32(47):5377-5387.

29. Farmer $\mathrm{H}$, et al. Targeting the DNA repair defect in BRCA mutant cells as a therapeutic strategy. Nature. 2005;434(7035):917-921.

30. Robson M, et al. Olaparib for metastatic breast cancer in patients with a germline BRCA mutation. N Engl JMed. 2017;377(6):523-533.

31. Friboulet L, et al. ERCC1 isoform expression and DNA repair in non-small-cell lung cancer. $N$ Engl JMed. 2013;368(12):1101-1110.

32. Touat $\mathrm{M}$, et al. DNA repair deficiency sensitizes lung cancer cells to $\mathrm{NAD}^{+}$biosynthesis blockade. J Clin Invest. 2018;128(4):1671-1687.

33. Sistigu A, et al. Cancer cell-autonomous contribution of type I interferon signaling to the efficacy of chemotherapy. Nat Med. 2014;20(11):1301-1309.

34. Lord CJ, Ashworth A. PARP inhibitors: synthetic lethality in the clinic. Science. 2017;355(6330):1152-1158.

35. Dréan A, et al. Modeling therapy resistance in BRCA1/2-mutant cancers. Mol Cancer Ther. 2017;16(9):2022-2034.

36. Pettitt SJ, et al. Genome-wide and high-density CRISPR-Cas9 screens identify point mutations in PARP1 causing PARP inhibitor resistance. Nat Commun. 2018;9(1):1849.

37. Krishnakumar R, Kraus WL. PARP-1 regulates chromatin structure and transcription through a KDM5B-dependent pathway. Mol Cell. 2010;39(5):736-749.

38. Abiko K, et al. IFN- $\gamma$ from lymphocytes induces PD-L1 expression and promotes progression of ovarian cancer. Br JCancer. 2015;112(9):1501-1509.

39. Leduc $C$, et al. TPF induction chemotherapy increases PD-L1 expression in tumour cells and immune cells in head and neck squamous cell carcinoma. ESMO Open. 2018;3(1):e000257.

40. Jiao $S$, et al. PARP ihibitor upregulates PD-L1 expression and enhances cancer-associated immunosuppression. Clin Cancer Res. 2017;23(14):3711-3720.

41. Wang K, Wang J, Wei F, Zhao N, Yang F, Ren X. Expression of TLR4 in non-small cell lung cancer is associated with PD-L1 and poor prognosis in patients receiving pulmonectomy. Front Immunol. 2017;8:456.

42. Loi S, et al. Prognostic and predictive value of tumor-infiltrating lymphocytes in a phase III randomized adjuvant breast cancer trial in node-positive breast cancer comparing the addition of docetaxel to doxorubicin with doxorubicin-based chemotherapy: BIG 02-98. J Clin Oncol. 2013;31(7):860-867.

43. Brambilla E, et al. Prognostic effect of tumor lymphocytic infiltration in resectable non-small-cell lung cancer. JClin Oncol. 2016;34(11):1223-1230.

44. Robillard L. Preclinical evaluation of the PARP inhibitor rucaparib in combination with PD-1 PD-L1 inhibition in a syngenic BRCA2 mutant ovarian cancer model. Proceedings of the AACR. 2017;58(pt A):3650. Accessed January 7, 2019.

45. Mouw KW, Goldberg MS, Konstantinopoulos PA, D'Andrea AD. DNA damage and repair biomarkers of immunotherapy response. Cancer Discov. 2017;7(7):675-693.

46. Melton DW, et al. Cells from ERCC1-deficient mice show increased genome instability and a reduced frequency of S-phase-dependent illegitimate chromosome exchange but a normal frequency of homologous recombination. JCell Sci. 1998;111 (pt 3):395-404.

47. Niedernhofer LJ, et al. The structure-specific endonuclease Ercc1-Xpf is required to resolve DNA interstrand cross-link-induced double-strand breaks. Mol Cell Biol. 2004;24(13):5776-5787.

48. Schwartz EK, Heyer WD. Processing of joint molecule intermediates by structure-selective endonucleases during homologous recombination in eukaryotes. Chromosoma. 2011;120(2):109-127.

49. Campbell JD, et al. Distinct patterns of somatic genome alterations in lung adenocarcinomas and squamous cell carcinomas. Nat Genet. 2016;48(6):607-616.

50. McCabe N, et al. Deficiency in the repair of DNA damage by homologous recombination and sensitivity to poly(ADP-ribose) polymerase inhibition. Cancer Res. 2006;66(16):8109-8115.

51. Williamson CT, et al. ATM deficiency sensitizes mantle cell lymphoma cells to poly(ADP-ribose) polymerase-1 inhibitors. Mol Cancer Ther. 2010;9(2):347-357.

52. Siddoo-Atwal C, Haas AL, Rosin MP. Elevation of interferon beta-inducible proteins in ataxia telangiectasia cells. Cancer Res. 1996;56(3):443-447.

53. Härtlova A, et al. DNA damage primes the type I interferon system via the cytosolic DNA sensor STING to promote anti-microbial innate immunity. Immunity. 2015;42(2):332-343.

54. Teo MY, et al. DNA damage response and repair gene alterations are associated with improved survival in patients with platinum-treated advanced urothelial carcinoma. Clin Cancer Res. 2017;23(14):3610-3618.

55. Gao D, et al. Cyclic GMP-AMP synthase is an innate immune sensor of HIV and other retroviruses. Science. 2013;341(6148):903-906.

56 . West AP, et al. Mitochondrial DNA stress primes the antiviral innate immune response. Nature. 2015;520(7548):553-557. 